\title{
Observable Surface Anomalies Preceding Simulated Isolated Convective Initiation ${ }^{\mathscr{O}}$
}

\author{
LuKe E. MADAUS AND GREgORY J. HAKIM \\ University of Washington, Seattle, Washington
}

(Manuscript received 18 September 2015, in final form 29 February 2016)

\begin{abstract}
Idealized ensemble simulations of isolated convective initiation (CI) are analyzed to identify storm-scale features in surface weather fields that precede initiation in a variety of background environments and the observations that would be needed to resolve these features. Precipitating storms are identified with an object-based method and composites of surface anomalies are generated for the variables of interest surrounding times and locations of initiation. Correlation length scales and anomaly magnitudes throughout the CI process are examined in detail with the latter comparing favorably to anomaly estimates obtained from previous observational and modeling studies. Negative temperature anomalies due to cloud shadowing are found to be the most prominent storm-scale feature prior to initiation. Significant spatial correlations are shown to extend from the surface throughout the boundary layer and even into the cloud-bearing layer once deep convective clouds become established. The findings are discussed in the context of data assimilation, particularly with respect to current assumptions about surface observation error. It is shown that, to resolve the storm-scale anomalies in these simulations, the minimum necessary temperature and wind observation densities would likely be limited by spatial correlation length scale while moisture and pressure observations are more limited by observation error.
\end{abstract}

\section{Introduction}

The prediction of exactly when and where precipitating convective storms will develop within an unstable air mass [i.e., convective initiation (CI)] remains a challenging problem. This is particularly true for the prediction of "airmass-type" thunderstorms that can develop within a broad region of instability without significant large-scale forcing or where local, boundary layer processes are the primary driver of CI. Numerous studies ascribe failure to skillfully predict storm-scale convective development to a lack of spatially and temporally dense observations (e.g., Stensrud and Fritsch 1994; Dabberdt and Schlatter 1996; Mass et al. 2002; Roebber et al. 2002; Fowle and Roebber 2003; Dabberdt et al. 2005; Gallus et al. 2005; Snook et al. 2015). Of

Supplemental information related to this paper is available at the Journals Online website: http://dx.doi.org/10.1175/MWR-D15-0332.s1.

Corresponding author address: Luke E. Madaus, Dept. of Atmospheric Sciences, University of Washington, Box 351640, Seattle, WA 98195.

E-mail:1madaus@atmos.washington.edu current observation platforms, only Doppler radars and some satellite products are able to produce observations on a temporal and spatial scale consistent with these requirements. As such, these platforms have received the majority of research attention. However, these observations provide limited utility in assessing the preconvective environment, essential for the prediction of CI.

Surface weather observations have received more limited attention for describing storm-scale features in the preconvective environment, chiefly due to their relatively poor spatial density (e.g., Mass et al. 2002; Sun et al. 2014). Recent studies have demonstrated that frequent, dense surface observations can contribute to improved CI forecasts (e.g., Liu and Xue 2008; Sobash and Stensrud 2015). To date, this forecast improvement has mostly been attributed to improved representation of broader mesoscale forcings (e.g., fronts or drylines) and reducing near-surface model biases (Sobash and Stensrud 2015). Here we wish to expand these findings by examining the potential for dense surface observations to describe structures on the scale of individual thunderstorms.

Surface observations are available at subhourly, kilometer-scale density or better in many locations from 
extant, yet underutilized networks such as private citizen weather stations (Madaus et al. 2014) and smartphone barometers (Mass and Madaus 2014). Exploiting these observations and enhancing existing surface observation networks may provide a storm-scale surface network capable of satisfying the requirements for CI forecasting. Yet, there has been little work to date describing how surface weather fields are represented in numerical simulations of CI, particularly for isolated convection where local, boundary layer processes are dictating the evolution. As such, there is little description of which surface variables are most relevant to observe, how sensitive these observations would need to be, or the requisite density of these observations in time and space. To assess these questions, the surface evolution of the CI process in modern, kilometer-scale numerical models must be better documented. This work uses idealized simulations of locally forced CI to investigate if there are observable storm-scale features in surface weather fields that precede isolated CI in light of current observation limitations.

The remainder of this paper is outlined as follows. A review of previous attempts to quantify the relationship between surface characteristics and convective initiation is given in section 2. Our methods, including the model configuration and analysis techniques, are given in section 3. The results are presented in section 4 with a discussion of the implications of these results in section 5 . We conclude by summarizing our findings and their limitations in section 6 .

\section{Background}

\section{a. Role of the surface in convective initiation}

The process of CI has typically been viewed in the parcel model context. Surface (or near surface) observations of atmospheric properties (temperature, moisture, wind, and pressure) are connected to the CI process from the parcel model in the following ways:

- Temperature-Relatively warm regions have greater free energy, and therefore an increased potential for positive buoyancy and reduced convective inhibition (CIN).

- Moisture-For parcels with the same temperature, moister rising parcels reach their lifted condensation level (LCL) at a lower altitude. Once the LCL is reached, latent heat release from condensation contributes to positive buoyancy and reduced CIN.

- Wind-Areas of convergence near the surface lead to upward vertical motion through mass conservation, sustaining rising parcels against CIN.

- Pressure-Areas of lower pressure near the surface can promote low-level convergence and can indicate rising motion above.
Characterizing the variability in these fields is crucial for successful CI forecasting. Near-surface temperature and moisture may vary for a number of reasons, but surface fluxes strongly influence local variability. Several studies note that surface heat and moisture flux variations can impact when and where convection will develop (e.g., Koch 1984; Yan and Anthes 1988; Childs et al. 2006; Holt et al. 2006). However, the relative importance of heat and moisture fluxes remains uncertain. Crook (1996) and Kang and Bryan (2011) find, through observation and idealized modeling studies, that local surface temperature variations exert stronger control over CI occurrence than moisture variability. However, other modeling studies based on specific cases (e.g., Droegemeier and Wilhelmson 1985; Clark et al. 2004; Martin and Xue 2006; Zhang et al. 2015) suggest that CI is most sensitive to moisture variability. Furthermore, Kang and Bryan (2011) note that with more homogeneous temperature distributions the variability in surface moisture plays a greater role in controlling CI occurrence. These studies collectively indicate that both temperature and moisture variability can potentially be important to capture for CI prediction. The relative importance of these variables has implications for which surface observations are more likely to discriminate locations of CI, and a better characterization of the magnitudes of variability in these surface fields relative to the potential observation error is needed.

There are additional ways for storm-scale variability in surface fields to locally enable or inhibit CI. Variations in cloudiness can create gradients in diabatic heating at the surface (and, consequently, surface temperature and moisture) due to cloud "shadowing" (Koch 1984; Lohou and Patton 2014; Rieck et al. 2015). Observational studies show that diabatic heating differences can change local pressure distributions, generating areas of increased convergence and vertical motion (e.g., Benjamin and Carlson 1986; Bluestein et al. 1988). Once convective storms develop, significant downdrafts due to evaporative cooling can contribute to cold pool formation at the surface, regardless of whether or not precipitation actually reaches the ground. Cold pool boundaries have frequently been examined as focal points for CI (e.g., Droegemeier and Wilhelmson 1985). Here we are more interested in quantifying the initial development of convection in a previously undisturbed environment, as subsequent forecasts of new initiation are likely affected by errors in the initial outbreak.

\section{b. Estimates of localized surface variability in the preconvective environment}

Observational studies suggest that meso- $\beta$ - and meso$\gamma$-scale variations in boundary layer fields preceding CI 
can be very subtle. Weckwerth et al. (1999) estimate that convergence due to only $1-2 \mathrm{~m} \mathrm{~s}^{-1}$ variability in winds may promote sustained upward motion. Arnott et al. (2006) observe convergent regions near the surface preceding $\mathrm{CI}$ as small as $2-5 \mathrm{~km}$ in extent during the International $\mathrm{H}_{2} \mathrm{O}$ Project (IHOP; Weckwerth and Parsons 2006). Using IHOP radar refractivity measurements, Fabry (2006) shows that moisture variability in the convective boundary layer (CBL) can be as small as $0.25 \mathrm{~kg} \mathrm{~m}^{-3}$ over a $5-\mathrm{km}$ length scale. Furthermore, model-based estimates suggest that local variations in moisture of as little as $1 \mathrm{~g} \mathrm{~kg}^{-1}$ are enough to support or discourage CI (Crook 1996; Martin and Xue 2006). Simulations suggest that observations on a spatial density of at least the CBL height are necessary to capture horizontal variability (Jonker et al. 1999). Evaluation of these sensitivities and variability in model simulations of $\mathrm{CI}$ is critical for evaluating the utility of a potential dense observing network.

\section{c. Scope of this study}

As noted above, it is well established that synoptic and broad mesoscale forcing (e.g., fronts, drylines, cold pool boundaries) can act as a focal point for CI (e.g., Owen 1966; Ziegler et al. 1997). Dense surface observations have already been shown to improve forecasts of broader mesoscale features (e.g., Wheatley and Stensrud 2010; Madaus et al. 2014), which has, in turn, led to more accurate CI forecasts (Sobash and Stensrud 2015). Therefore, the practical predictability of CI (and the near-surface properties of the preconvective environment) can immediately be enhanced by more accurate analyses of these features. Here, we wish to examine the surface storm-scale variability in the preconvective environment absent these effects. While it is unlikely that CI in the real atmosphere will be completely removed from larger-scale effects, this underlying storm-scale variability still exists and can play a critical role in dictating CI; here we will examine that contribution to $\mathrm{CI}$ in detail.

More generally, studies have repeatedly demonstrated that small, storm-scale forecast errors surrounding CI can rapidly propagate upscale, limiting predictability to a matter of hours (e.g., Brooks et al. 1992; Zhang et al. 2003; Hohenegger and Schaar 2007). Though the downscale propagation of small errors on broad meso- and synoptic scales also constrains and ultimately limits CI predictability (e.g., Durran and Weyn 2016; Torn and Romine 2015), for short-term CI forecasts (less than $6 \mathrm{~h}$ ) stormscale errors have great importance. Therefore, this study numerically simulates idealized, isolated $\mathrm{CI}$ in the absence of prescribed dynamic forcing to examine how surface observations might constrain these errors, and what quality and quantity of observations might be required.

\section{Methods}

\section{a. Convective environments}

To examine $\mathrm{CI}$ in idealized simulations without imposing a large-scale forcing mechanism, it is necessary to choose appropriate environments. Specifically, we seek realistic thermodynamic environments where CI may occur only due to local, boundary layer processes. A total of 23 observed soundings are subjectively chosen to provide environmental conditions in our simulations (Fig. 1). These soundings span the continental United States east of the Rocky Mountains during JuneOctober 2014. Synoptic charts, radar data, and storm reports are scrutinized to identify times and locations where

- precipitating convection developed within $12 \mathrm{~h}$ after the sounding time, and

- large-scale forcing (e.g., fronts, upper-level shortwaves) was distant or appeared absent from the event.

The soundings at locations shown in Fig. 1 cover a variety of warm-season environments in the United States. Figure 2a shows the soundings from three particular environments that will be discussed in greater detail later in this paper. (Soundings from all environments used in this work are shown in Fig. S1 in the online supplemental material.) Figure $2 b$ shows the surfacebased convective available potential energy (CAPE) and CIN computed from these soundings, both for the initial time and the forecast surface-based values based on the original sounding. Initial CAPE ranges over $0-2500 \mathrm{~J} \mathrm{~kg}^{-1}$ and CIN over $0-600 \mathrm{~J} \mathrm{~kg}^{-1}$. The forecasted surface-based values show CIN reduced to zero or near zero in all but one environment as the day evolves, permitting CI to occur. We expect that the environmental diversity captured by these soundings provides an estimate of the variability and broader applicability of the findings. Two of the environments provided by these soundings failed to produce deep convection in our simulations (including the one with forecast surfacebased CIN of $120 \mathrm{~J} \mathrm{~kg}^{-1}$ in Fig. 2b); we will discuss these environments further at the beginning of section 4 .

\section{b. Forecast model}

For idealized simulations, we use Cloud Model 1, revision 17 (CM1; Bryan and Fritsch 2002), which has enjoyed widespread use in the severe storms community. From each sounding identified in Fig. 1, homogeneous initial conditions are generated for a $104 \mathrm{~km} \times 104 \mathrm{~km}$ domain. There is no topography included. Land surface properties are uniform and match the U.S. Geological Survey (USGS) land-use category that is most prevalent within a $100-\mathrm{km}$ radius 


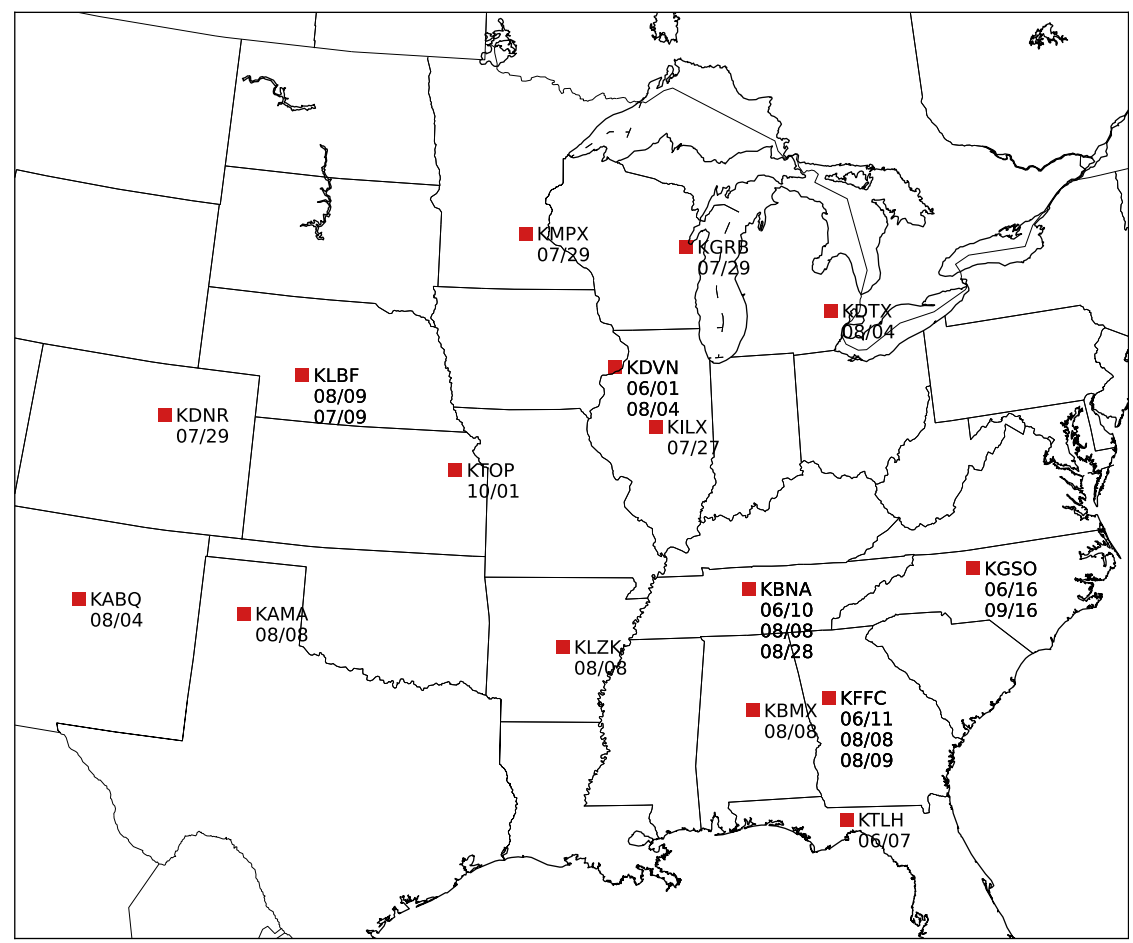

FIG. 1. Locations and dates of the soundings chosen as initial conditions for simulations. Soundings from 1200 UTC on each date are used. All soundings are from the year 2014.

of the sounding site. Boundary conditions are doubly periodic and the only external forcing is diurnal radiation. Simulations are run for $15 \mathrm{~h}$ from the time of the sounding launch.

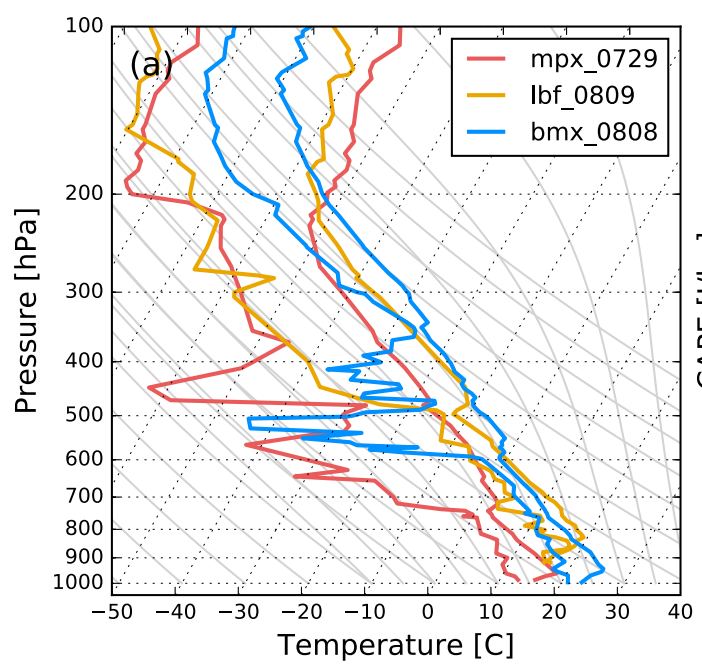

In many idealized convective simulations, deep convection is "forced" to initiate by the inclusion of some storm-scale perturbation, often a "warm bubble" or prescribed convergence. Here we wish to examine a free

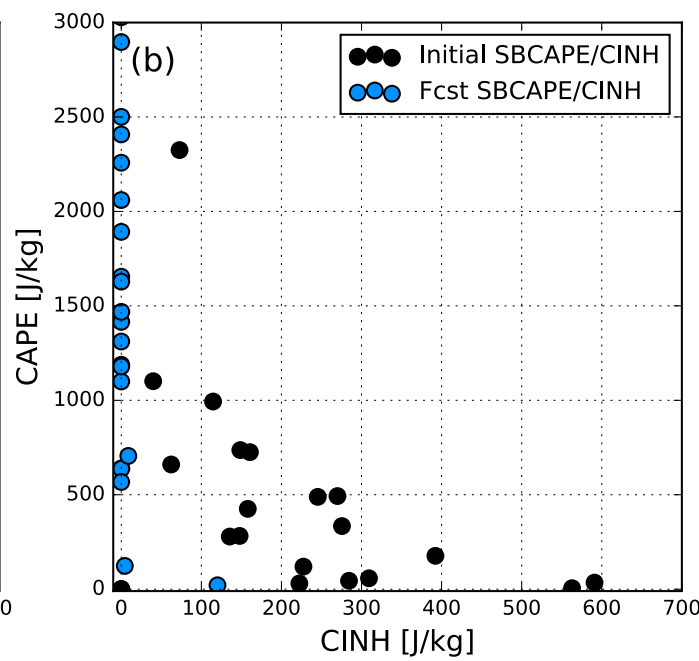

FIG. 2. (a) Temperature and moisture profiles from three specific soundings (KMPX 1200 UTC 29 Jul 2014, mpx 0729; KLBF 1200 UTC 9 Aug 2014, lbf_0809; and KBMX 1200 UTC 8 Aug 2014, bmx_0808) that will be referred to in more detail throughout this study. (b) CIN $\left(\mathrm{J} \mathrm{kg}^{-1}\right)$ and CAPE $\left(\mathrm{J} \mathrm{kg}^{-1}\right)$ for a surface-based parcel at both the initial sounding time (black circles) and for a forecast surface parcel in the initial sounding (blue circles) for all environments. 
process of CI without prescribing an initial lifting mechanism. In these simulations, we only impose random, white-noise, gridpoint perturbations to the initial potential temperature field at all model levels with a maximum magnitude of $0.2 \mathrm{~K}$ to promote random spatial variability. We experimented with different initial maximum perturbation magnitudes ranging from 0.05 to $0.5 \mathrm{~K}$, but the results were insensitive to this magnitude. The environments chosen have sufficient conditional instability that convection initiates on its own after diurnal heating and convective mixing have developed a sufficiently deep convective boundary layer. In each environment examined, the first convective clouds appear between 2 and $8 \mathrm{~h}$ after the beginning of the simulation and are uncorrelated with the initial noise perturbations. While this "unforced" CI method limits the possible environments that can be evaluated, these simulations allow assessment of the CI process absent a prescribed, forced initiation.

For each sounding environment, a 25-member ensemble is produced at 1-km horizontal grid spacing. Ensemble diversity is solely due to different initial random potential temperature perturbations. There is debate over the horizontal resolution required to adequately simulate deep convection (e.g., Mass et al. 2002; Bryan et al. 2003; VandenBerg et al. 2014). While higher resolution $[O(100) \mathrm{m}]$ may be required to adequately simulate boundary layer convective rolls common to conditionally unstable environments (Weckwerth et al. 2014; Nowotarski et al. 2014), 1-km grid spacing is chosen as it can provide acceptable forecasts of individual convective storms and more closely matches what current and near-future operational forecasting systems may hope to achieve (e.g., Skamarock 2004; Schwartz et al. 2009; VandenBerg et al. 2014). Future studies may wish to examine simulations at multiple resolutions to compare how the scale and magnitude of observed features may change going from realistic, observation-like (higher resolution) to operational forecasts (lower resolution).

Model vertical grid spacing is $80 \mathrm{~m}$ in the lowest $3.2 \mathrm{~km}$ and linearly increases to $500 \mathrm{~m}$ from $9 \mathrm{~km}$ to the model top at $18 \mathrm{~km}$. Rayleigh damping of vertical motions is applied above $15 \mathrm{~km}$. Parameterizations used include the Yonsei University (YSU) planetary boundary layer (PBL) scheme (Hong et al. 2006) and the NASA Goddard longwave and shortwave radiation (Chou and Suarez 1999, 2001). The NASA Goddard Lin-FarleyOrville (LFO) microphysics scheme is used, as in the CM1 model the radiation scheme is specifically designed to be used with this microphysics scheme.

The land surface scheme used in these simulations is CM1's sfcmodel = 2, which is adapted from the Weather
Research and Forecasting (WRF) similarity theory scheme and includes a thermal diffusion soil model (Skamarock et al. 2008). The skin temperature is prognostic in this model but soil moisture availability is held fixed over time and is based on the land-usage category as described above. The skin temperature and deeplayer soil temperature are set to the surface temperature from the original environmental sounding. We experimented with varying the deep-layer soil temperature value by up to $5 \mathrm{~K}$ from the surface temperature, but this did not appear to change our results.

\section{c. Analysis methods}

\section{1) COMPOSITING ANALYSIS}

To examine the typical characteristics of surface fields surrounding the CI process, we employ a compositing method centered on times and locations where CI occurs. To determine these times and locations, we use an objectbased method of identifying and tracking cloud and storm objects loosely based on the Method for Object-based Diagnostic Evaluation (MODE; Davis et al. 2006). Specifically, for each ensemble member forecast, the simulated composite reflectivity field (CREF) is scrutinized at 5-min intervals to find areas where CREF $>20 \mathrm{dBZ}$. While this isolates candidate storm "objects" from the background, additional processing is needed to separate storm objects in close proximity. Within each identified area, all local maxima of CREF are identified and, if there is more than one, a watershed algorithm is applied to separate the region into unique objects surrounding each maxima. All objects in this set with a spatial area less than $9 \mathrm{~km}^{2}$ are discarded. The remaining objects are considered to be the set of deep "clouds" present in the model at that time.

The weighted centroids of these CREF cloud objects are tracked through time using the Python TrackPy package (Allan et al. 2014) at 5-min intervals. Only objects successfully tracked for at least $20 \mathrm{~min}$ are considered candidate storms. At each time, we examine the accumulated surface precipitation field within the boundaries of each cloud object. The cloud object is labeled a "precipitating storm" if the precipitation has increased within the object boundaries since the previous time. We identify times and locations where CI occurs by noting the time and centroid of any object when and where precipitation first reaches the ground in that object. We find that this definition of CI, requiring a minimum CREF of $20 \mathrm{dBZ}$ and observed precipitation at the ground, provides consistent and reliable detection of storm objects from simulations of different environments. Furthermore, as will be shown in section 4 , the time when precipitation is first observed at the ground 
coincides with a fundamental change in the patterns observed in the surface fields, marking a natural differentiator between pre- and postinitiation periods.

For our compositing analysis, we select the first four precipitating storm objects that form in each ensemble member. Preliminary analysis showed that storms that form later in the simulations tended to form along the edges of cold pools produced by earlier storms, and the signals of these cold pools dominated our analysis of preconvective surface features. While cold pools are an important potential focal point for CI, surface observations have already been seen to improve cold pool representation in models (e.g., Wheatley and Stensrud 2010; Dong et al. 2011). As noted in section 2, here we want to focus on surface features surrounding the initial wave of CI in the model, absent more organized forcing. With 25-member ensembles, selecting the first four precipitating storms in each member gives a sample size of 100 storms in each composite, for each sounding environment.

To create composites, for each storm the entire doubly periodic domain is translated to center on the time and location when CI occurred (by our definition above). Anomalies are computed in the fields of interest at that time by subtracting the domainwide horizontal mean of those fields in the full simulation domain. This process is repeated at 5-min intervals for times up to $180 \mathrm{~min}$ before and $60 \mathrm{~min}$ after CI, with the domain remaining centered on the location of CI. No accommodation is made for variations in storm motion vectors; for the initial wave of CI the storm motion from the cells examined is generally parallel. The anomaly fields from all storms at each time relative to $\mathrm{CI}$ are then averaged together to form a composite. The sample size of storms (100) is large enough that the signatures of any additional storms surrounding each contributor to the composite mostly cancel out. An analysis of these composites follows in sections $4 \mathrm{~b}$ and $4 \mathrm{c}$.

\section{2) Correlation LeNGTH SCALES}

When developing an observing network to sample the preconvective environment, it is important to consider the average spatial extent of the impact for a given observation, as this has implications for the necessary observation density. To investigate this, average correlation length scales for relevant surface variables are computed for each environment's ensemble simulation. Specifically, for a given variable, at each point in the domain the ensemble correlation of that point to each other point in the doubly periodic domain (of the same variable) is computed. The correlation magnitude is then binned by distance from that point to the nearest kilometer, and a binwise average is computed, again for each point. By averaging this correlation-by-distance profile across all points at each time in the simulation, the spatial scale of correlation at a given time in the simulation may be examined. A discussion of the results of this analysis is given in section $4 \mathrm{~d}$.

In addition to horizontal correlation length scales, average vertical correlations as a function of time are also computed for selected variables. These correlations illustrate the potential impact of a surface observation on atmospheric properties aloft. We discuss one such correlation analysis in section 4e.

\section{Results}

\section{a. CI simulation evolution and comparisons to $C I$ "failure" environments}

In the ensemble simulations for all environments, the boundary layer depth is observed to grow throughout the first several hours of integration in response to increasing heat flux from the surface as solar radiation increases. For most environments simulated, cloud formation is observed between 2 and $8 \mathrm{~h}$ into the simulations, with precipitation first occurring between 5 and $13 \mathrm{~h}$ into the simulations (Fig. 3; squares). For all but two of the environments, the deepest convective clouds reach the tropopause level inferred from the initial environment sounding, indicating that deep convection occurs.

For the remaining two environments (9 July 2014 KLBF and 29 July 2014 KMPX; profiles shown in Fig. 2a), deep CI does not occur in the simulations. In the KLBF environment, the simulations produce scattered shallow cumulus clouds but no precipitation at the surface, and it was noted from Fig. $2 b$ that this environment had nonzero forecast surface-based CINH. In the KMPX environment, shallow-to-towering cumulus clouds are produced but, though some of these clouds do produce weak precipitation, the size and duration of tracked reflectivity objects in most of the ensemble members does not meet the requirements for storm objects with successful CI outlined in section 3c. Nevertheless, these two environments provide examples of CI failure to compare with successful CI environments.

We briefly consider the differences between the evolution of the two CI failure environments and the remaining 21 successful CI environments with respect to variability in temperature at the surface. Figure 3 shows the ensemble-averaged spatial variance in the simulated 2-m temperature fields as a function of time for all the environments simulated. The two CI failure environments are highlighted in red and gold. The variance in the surface temperature field, while initially small, begins increasing once clouds are present in the domain for 


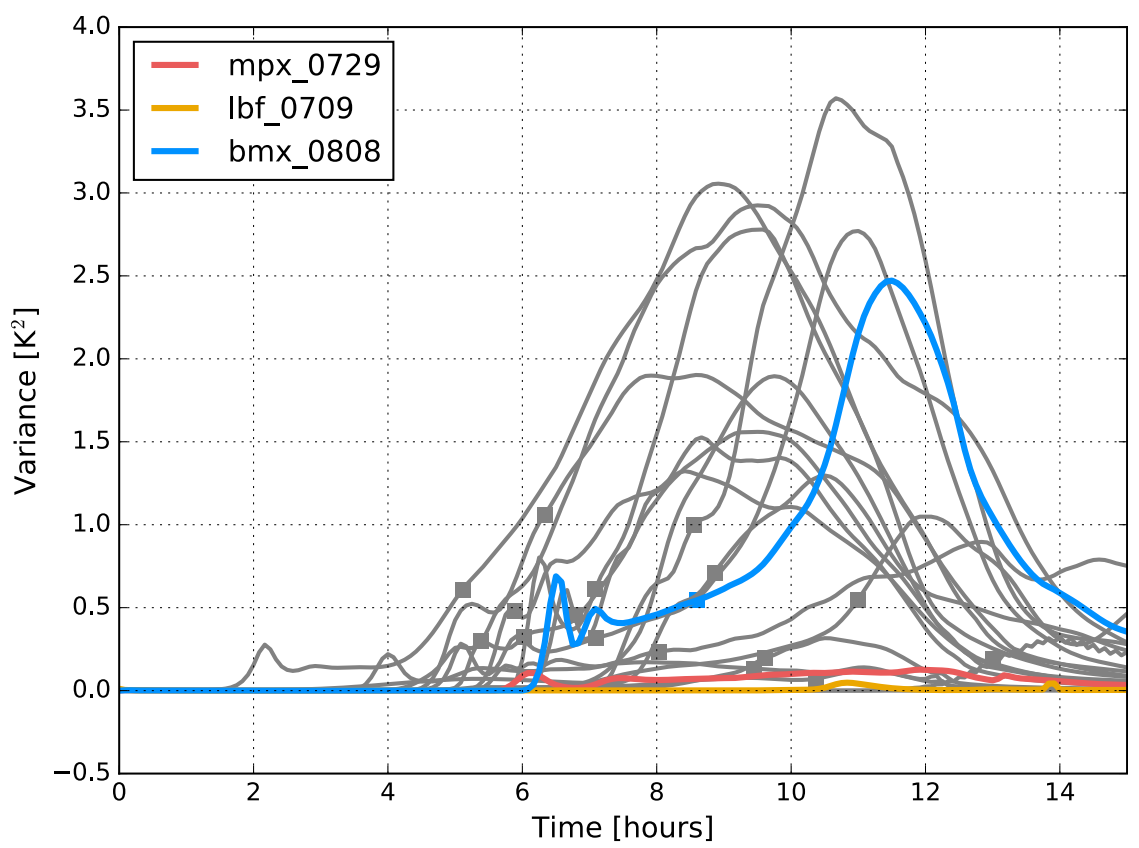

FIG. 3. Ensemble-averaged spatial variance in the 2-m temperature field as a function of time for all environments simulated. The ensemble-averaged time when precipitation is first observed at the surface for each environment is shown by squares. The two CI failure environments (mpx_0729, red; and lbf_0709, gold) are highlighted, as well as the 8 Aug 2014 KBMX environment (bmx_0808; blue). All other environments simulated are shown in gray.

reasons that will be described in the following sections. This variance grows as convection deepens and matures, being augmented by the development of cold pools once precipitation has begun. Variance in the timing of precipitation (and, consequently, cold pool) development between ensemble members may contribute somewhat to a rapid increase in 2-m temperature variance in addition to horizontal inhomogeneity. However, for most environments, the time of precipitation onset in various ensemble members differed from the mean by no more than $10 \mathrm{~min}$. The variance reduces toward the ends of the simulations as the environment stabilizes and active convection diminishes. The magnitude of the variance differs between environments, depending on the number of clouds that form and the depth of the convective clouds.

It is encouraging to see in Fig. 3 that many of the environments where deep CI occurs show an hour or more of increasing spatial variance in 2-m temperature prior to the onset of precipitation (shown by squares), suggesting a window prior to CI where surface observations may operate to help constrain the variability. In contrast to this variance growth in deep CI simulations, the two CI failure simulations with shallower clouds have relatively low spatial variance in surface temperature throughout their evolution. While it appears that deep convective clouds can be associated with greater surface temperature variability, there are some environments where deep CI does occur, based on our criteria, which have similar variance magnitudes to the CI failure simulations for extended periods. Thus, while it seems likely that a dense surface observing network will be able to contribute to constraining robust, deep CI, such a network may not be sufficient to separate CI success and failure for more marginal cases.

Furthermore, in our experiment setup, the background environment is tightly constrained without large-scale variability. Therefore, it is difficult with these experiments to address how surface observations may constrain broader variability, particularly above the boundary layer, for processes like entrainment that are likely promoting or discouraging CI in the real world. For the remainder of our analysis we will focus specifically on the storm-scale variability surrounding those storms that do develop. As such, the two CI failure environments will not be included in the subsequent analysis.

\section{b. Compositing analysis}

We now focus on specific features in surface fields surrounding developing storm objects by examining the CI-relative composites of surface anomalies. Figure 4 

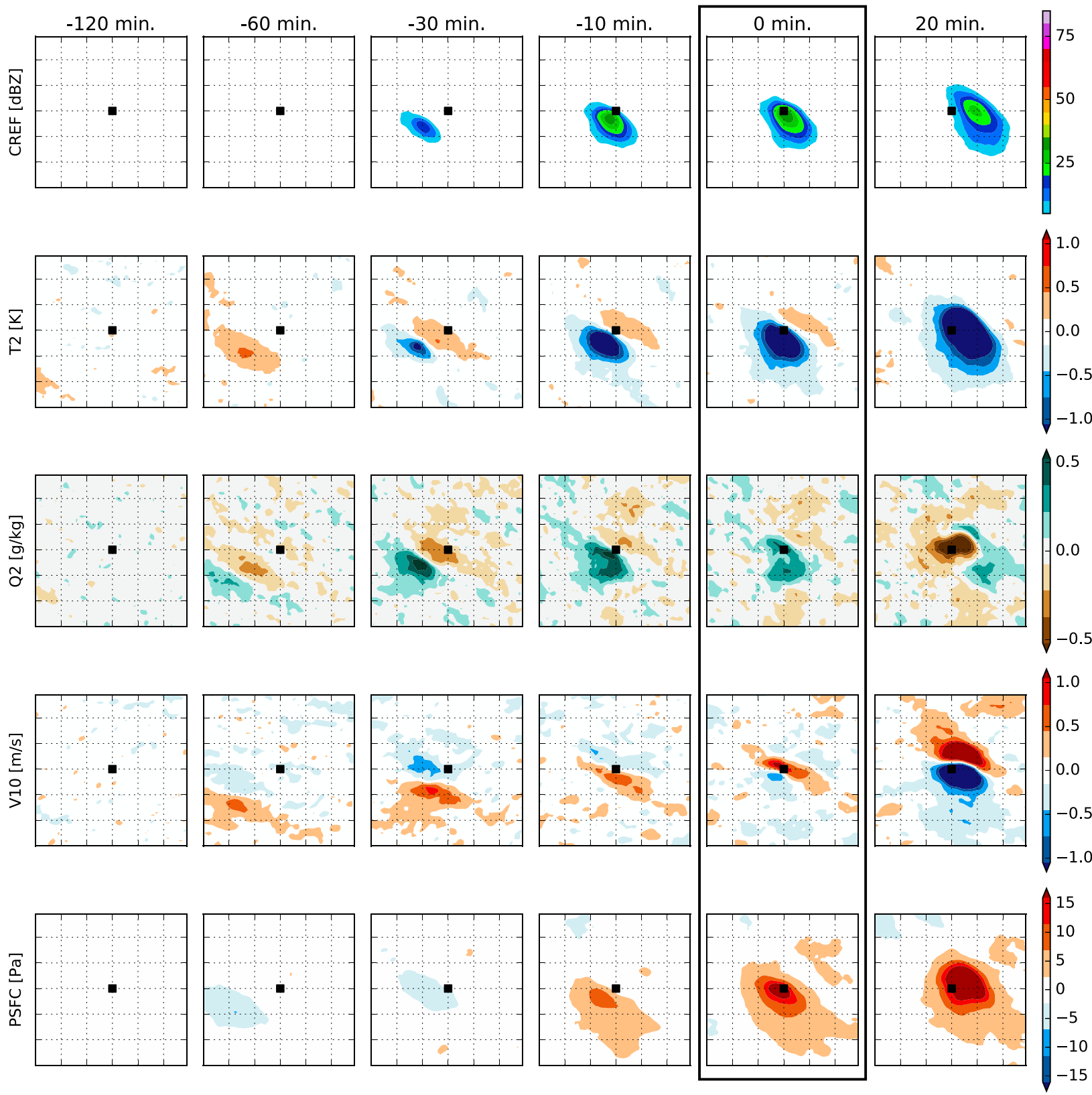

FIG. 4. Composited anomalies from the 8 Aug 2014 KBMX environment simulations relative to the location (black squares) and time (columns) of CI for (from top to bottom) composite reflectivity [CREF (dBZ)], 2-m temperature [T2 (K)], 2-m mixing ratio [Q2 (g kg $\left.\left.{ }^{-1}\right)\right]$, $10-\mathrm{m} v$-wind component [V10 $\left.\left(\mathrm{m} \mathrm{s}^{-1}\right)\right]$, and surface pressure [PSFC $\left.(\mathrm{Pa})\right]$. The column at the time of CI $(0 \mathrm{~min})$ is boxed. For scale, the gray dashed grid shows $10 \mathrm{~km}^{2}$ squares. Though the composite anomalies are generated using the entire simulation domain, only the $60 \times$ $60 \mathrm{~km}^{2}$ region surrounding the location of initiation is shown for clarity.

shows an example of the CI-relative composites of composite reflectivity (CREF), 2-m temperature (T2), 2-m mixing ratio (Q2), 10-m $v$-wind component (V10), and surface pressure (PSFC) for the 8 August 2014 KBMX environment (highlighted in Figs. 2 and 3). The composites from this environment exemplify the salient features seen in composites from the other environments. Below is a description of these features for each variable. Discussion of the magnitudes of these features follows in section $4 \mathrm{c}$.

\section{1) Composite ReFlectivity}

The CREF composites in Fig. 4 (top row) illustrate the reliability of the object-based compositing technique. No signal is present until approximately $30-40 \mathrm{~min}$ before CI (again, here defined as the onset of precipitation), after 
which a smoothed CREF maximum grows, passing over the location of CI (black square) at the time of CI ( $0 \mathrm{~min}$, fifth column) before moving off. This affirms that the composite is reliably collocating simulated storm objects.

\section{2) Surface temperature}

In composite 2-m temperature (T2) (Fig. 4, second row), a warm anomaly appears as early as $120 \mathrm{~min}$ before CI for this particular environment; in other environments the time of the first discernible warm anomaly varies from 90 to $170 \mathrm{~min}$ prior to $\mathrm{CI}$. The ultimate source for this anomaly varies across environments. In some environments, the deepening CBL organizes into horizontal convective rolls (Weckwerth et al. 1999) with alternating warm and cold anomalies collocated with rising and sinking branches of these rolls. Stochastic variability can also promote anomalously warm temperatures in certain regions.

In simulation of environments like KBMX in Fig. 4, cumulus development prior to the formation of precipitating storms also contributes to surface temperature variability. This is suggested in the horizontal T2 variance evolution (Fig. 3; blue line) where T2 variance grows from the first appearance of clouds (at $6 \mathrm{~h}$ ) through the average time of CI (at $8.5 \mathrm{~h}$ ). Given the presence of clouds $2.5 \mathrm{~h}$ prior to $\mathrm{CI}$, the deep precipitating clouds, that are the components of these composites, form within a preexisting cloud field. The left column of Fig. 5 shows that the warm T2 anomaly present $60 \mathrm{~min}$ prior to CI (T2; bottom row) is collocated with a relative minimum in the surrounding cloud field, shown in the composited column-integrated cloud water (CLOUD; top row). The lack of deep clouds yields a positive downward shortwave radiation anomaly at the surface (RADSW) and corresponding upward heat flux anomaly (HFX) that promote the positive T2 anomaly. This composited warm anomaly propagates toward the location of CI, attaining a maximum magnitude between 45 and $60 \mathrm{~min}$ before CI. The anomaly area greater than $0.1 \mathrm{~K}$ is broad, extending some $30 \mathrm{~km}$ in its widest dimension, but the area of $>0.5 \mathrm{~K}$ is very localized-only $2 \mathrm{~km}$ in diameter.

Approximately $30 \mathrm{~min}$ prior to CI, a cold anomaly develops, collocated with the CREF maximum. Examination of the surface heat fluxes and column cloud water (Fig. 5) relates this anomaly to cloud shadowing: the reduction of incoming shortwave radiation at the surface due to the cloud overhead. In Fig. 5, the composite column-integrated frozen and liquid water (CLOUD; first row) shows a distinct cloud object approximately $40 \mathrm{~min}$ prior to CI. This cloud object is collocated with a negative shortwave radiation anomaly at the surface (RADSW), reduced upward heat flux at the surface (HFX) and the negative T2 anomaly described above. These anomalies grow and propagate in tandem as the cloud object evolves toward initiation. A cloud-shadowed T2 anomaly is observed in all of the simulated environments. We note that since the NASA Goddard radiation scheme uses a two-stream approximation (where only the vertical component of radiation is evaluated, regardless of the solar zenith angle), this cloud shadow always occurs directly underneath the developing cloud. Further discussion of the cloudshadowing effect follows in the discussion of Fig. 6 and with respect to vertical correlations in section $4 \mathrm{e}$.

In the $30 \mathrm{~min}$ prior to $\mathrm{CI}$, the cloud-shadowed cool temperature anomaly grows in size while the warm anomaly reduces in both magnitude and extent. After precipitation begins, the area of the cold anomaly rapidly expands and the magnitude continues to increase. However, at this point the cloud shadowing is augmented by cool downdraft air descending in the nowprecipitating storm and this cold anomaly could be better characterized as a cold pool.

\section{3) Surface MOISTURE}

Anomalies in the surface moisture field (Fig. 4; Q2) do not become noticeable until about $60 \mathrm{~min}$ prior to $\mathrm{CI}$. A distinct dipole structure is apparent during the hour before precipitation begins, with a negative moisture anomaly preceding a positive anomaly with respect to their motion. The center of the dipole tends to remain directly underneath the cloud object (cf. CREF in top row) with the positive moisture anomaly collocated with the negative temperature anomaly at $30 \mathrm{~min}$ prior to $\mathrm{CI}$. The warm anomaly at the surface is associated with locally enhanced PBL mixing, which contributes to reduced moisture in the PBL and a negative Q2 anomaly. In contrast, locally reduced sensible heat flux in the cloud-shadowed region weakens PBL mixing, which results in higher moisture in that area. The moisture anomaly is slightly smaller in extent than the temperature anomaly, but the area of $>0.1 \mathrm{~g} \mathrm{~kg}^{-1}$ still becomes $20 \mathrm{~km}$ across at its widest dimension in the $30 \mathrm{~min}$ prior to CI. After CI, the positive moisture anomaly is quickly replaced by an expanding negative anomaly as the cold pool develops. While the air in the developing cold pool is nearly saturated, the saturation mixing ratio in the cold pool is lower than the ambient environmental mixing ratio, yielding a negative anomaly.

\section{4) 10-M WINDS}

Near-surface winds can be considered in vector form (both $u$ - and $v$-wind components) or by examining each component individually; here we select the $v$-wind component (V10) as it showed the strongest signal given 

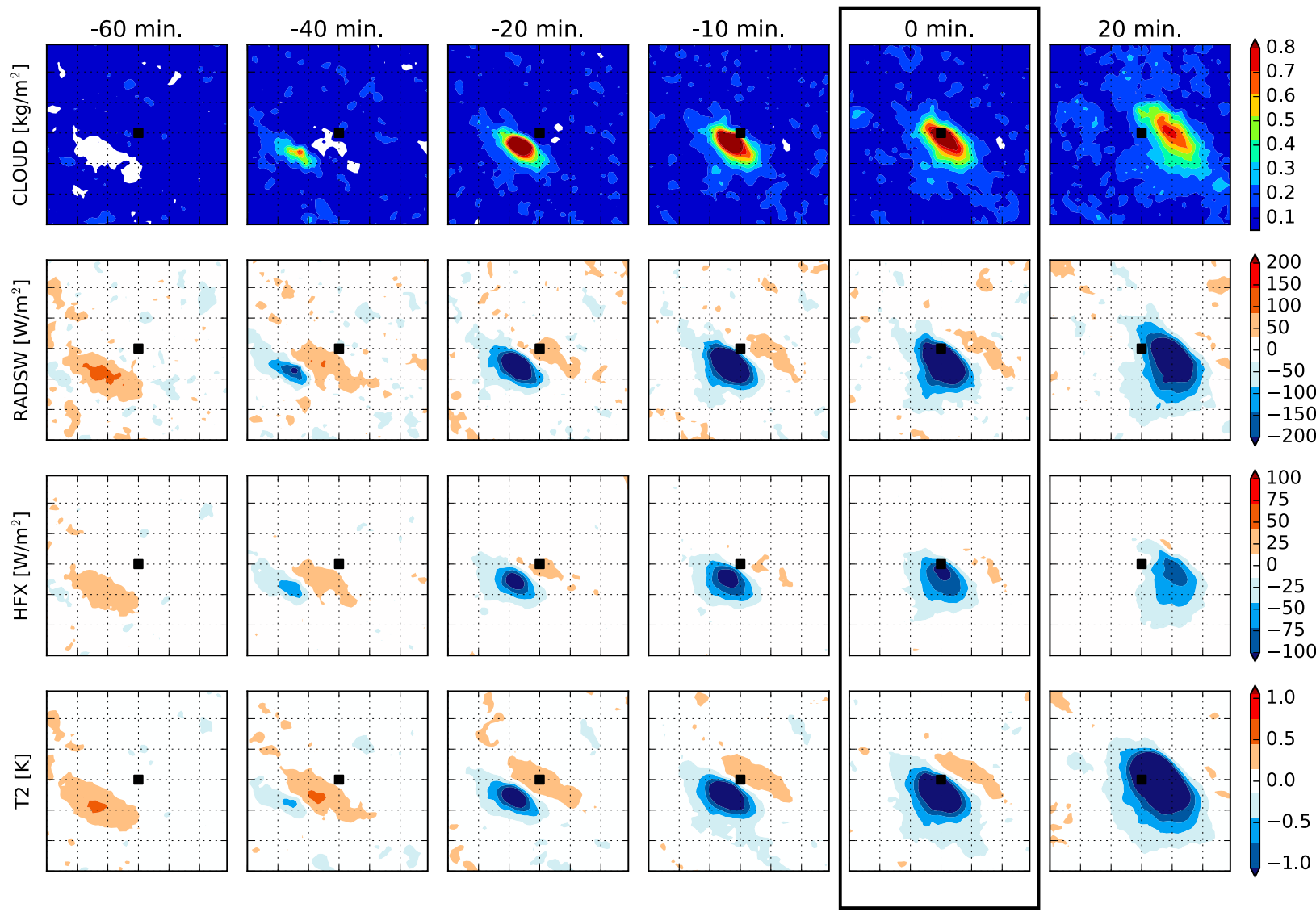

FIG. 5. Composited features from the 8 Aug 2014 KBMX environment simulations relative to the location (black squares) and time (columns) of CI for (from top to bottom) column-integrated frozen and liquid water [CLOUD ( $\left.\mathrm{kg} \mathrm{m}^{-2}\right)$ ], shortwave radiation flux anomaly at the surface [RADSW $\left(\mathrm{W} \mathrm{m}^{-2}\right)$ ], upward surface heat flux anomaly [HFX $\left.\left(\mathrm{W} \mathrm{m}^{-2}\right)\right]$, and 2-m temperature [T2 $\left.(\mathrm{K})\right]$. The column at the time of CI $(0 \mathrm{~min})$ is boxed. For scale, the gray dashed grid shows $10 \mathrm{~km}^{2}$ squares. Though the composite anomalies are generated using the entire simulation domain, only the $60 \times 60 \mathrm{~km}^{2}$ region surrounding the location of initiation is shown for clarity.

the background wind profile of the 8 August 2014 KBMX environment and for ease of comparison with the other variables shown. Note that the mean storm motion has not been subtracted from these winds. The expected signature of low-level convergence first becomes apparent by $60 \mathrm{~min}$ prior to $\mathrm{CI}$. The convergence strengthens through the following $30 \mathrm{~min}$, with the $v$ extrema separated by $7 \mathrm{~km}$, on average. Immediately prior to CI, the convergent anomalies weaken and are rapidly replaced by diverging wind anomalies. These diverging winds again represent cold pool generation with the now-precipitating downdraft spreading out as it reaches the surface.

\section{5) SuRfaCE PRESSURE}

The surface pressure (PSFC) anomalies compliment the $10-\mathrm{m}$ wind and 2-m temperature anomalies in the hour leading up to CI. A negative pressure anomaly is apparent beginning at $60 \mathrm{~min}$ prior to $\mathrm{CI}$ and is visible through $20 \mathrm{~min}$ prior to CI (not shown), consistent with the concurrent positive temperature anomaly and convergent winds. By $10 \mathrm{~min}$ prior to $\mathrm{CI}$, the negative anomaly has been replaced with a growing positive anomaly, likely in response to a developing downdraft aloft (as pressure responds to the integrated mass above a point) and the persistent cooling due to cloud shadowing. This positive anomaly grows rapidly following initiation-another symptom of cold pool development.

\section{c. Composite anomaly magnitudes}

The above descriptions (particularly the magnitudes and scales) apply specifically to the 8 August 2014 KBMX environment, but the analyses are performed on all environments and show similar evolution. Figure 6 shows time series of the maximum and minimum anomaly magnitude in the entire composited domain for the variables described above (except for CREF) and for each environment simulated. 

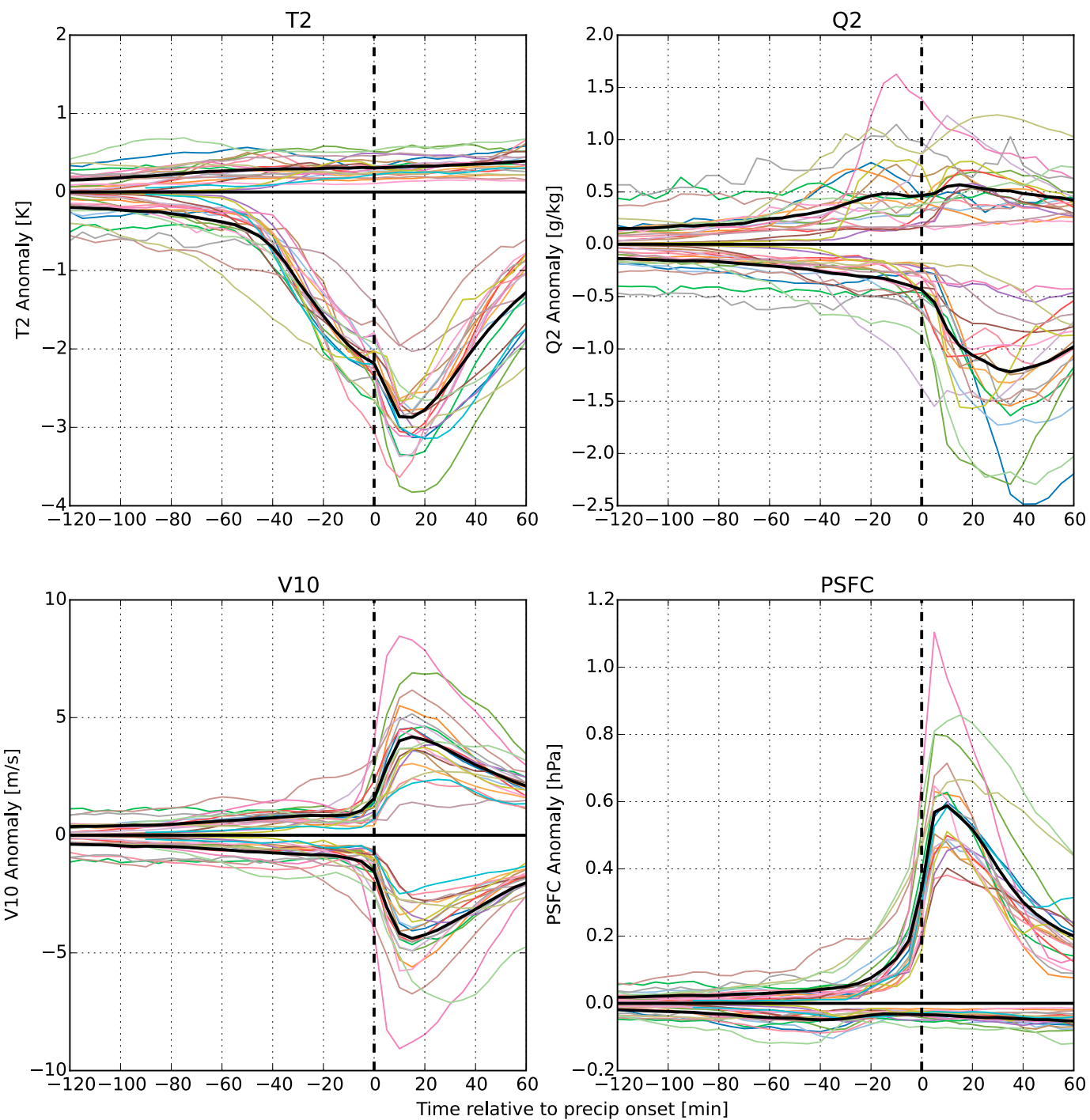

\begin{tabular}{|c|c|c|c|c|c|c|c|}
\hline & 0808 & & & 29 & 22 & top & \\
\hline & ilx & am & $d t x$ & $a b q$ & 729 & $28-$ & lbf \\
\hline 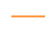 & ffc_0809 - & gso_0616 - & bna_0610 - & dvn_0601 - & tlh_0607 & dvn_0804 - & ffc_0611 \\
\hline
\end{tabular}

FIG. 6. Maximum and minimum composite anomaly magnitudes as a function of time relative to precipitation onset (CI) for 2-m temperature (T2), 2-m mixing ratio (Q2), 10-m $v$-wind component (V10), and surface pressure (PSFC) for each simulated environment (colors). The solid black lines for each panel show the mean maximum and minimum anomaly magnitude across all environments.

For T2 (Fig. 6, top left), the positive anomalies reach a mean magnitude of $0.3 \mathrm{~K}$, which, on an environment-byenvironment basis, generally remains larger than the mean negative magnitude until $40-60 \mathrm{~min}$ prior to CI. During this time, the cloud-shadowing effect becomes apparent in all environments, generating increasingly negative temperature anomalies to about $2 \mathrm{~K}$ in magnitude. From observations, Lohou and Patton (2014) note negative 2-m air temperature anomalies of about $1-\mathrm{K}$ magnitude and skin surface temperatures anomalies of up to $4 \mathrm{~K}$ in association with the passage of shallow cumulus clouds. Thus, the cloud-shadowed cold temperature anomalies simulated here appear reasonable, particularly for deeper convective clouds. A slight kink in the negative $\mathrm{T} 2$ temperature anomalies at the time of CI highlights the transition from cloud shadowing to cold pool generation as the primary source of the anomalies.

Surface humidity (Q2) has much more variability between environments. A general increase in both the 
positive and negative anomaly magnitude over time follows the strengthening dipole as shown in Fig. 4. The mean anomaly magnitudes increase from 0.1 to $0.5 \mathrm{~g} \mathrm{~kg}^{-1}$ during the $2 \mathrm{~h}$ prior to CI. In some environments, there are rapid increases in the positive or negative moisture anomaly beginning $30-40 \mathrm{~min}$ before CI. The maximum positive moisture anomaly during the $30 \mathrm{~min}$ prior to CI is well correlated $(r=0.54, p=0.01)$ to the ensemble mean, domain-averaged LCL in each environment $30 \mathrm{~min}$ prior to CI (Fig. 7). We note that the high confidence in this correlation comes mostly due to one environment (KDNR 29 July 2014) with a particularly high LCL height and maximum pre-CI moisture anomaly magnitude. Removing this environment still yields a positive correlation $(r=0.22)$, but the correlation is no longer statistically significant. Regardless, a positive correlation suggests that in environments with higher LCLs, a larger moisture anomaly is required for successful CI, agreeing with the parcel model. Negative moisture anomalies become more dominant after CI, but with a great deal of variance as the moisture properties of the cold pool are highly dependent on the environment throughout the depth of the precipitating cloud.

For surface winds (V10), another slow increase in both positive and negative anomaly magnitude is apparent throughout the $2 \mathrm{~h}$ prior to CI. These positive and negative anomalies as shown here do not necessarily imply convergence by themselves; however, an analysis of each environment's anomalies indicates that this is indeed the case. The average positive and negative anomalies are generally symmetric for each environment, reaching an average magnitude of $\pm 1 \mathrm{~m} \mathrm{~s}^{-1}$ from $-40 \mathrm{~min}$ through the time of CI. A rapid increase in anomaly magnitude after CI (to $\pm 4 \mathrm{~m} \mathrm{~s}^{-1}$ on average) shows the strong divergence with cold pool development.

Surface pressure anomalies remain very weak until $30 \mathrm{~min}$ prior to CI, with a mean amplitude of $\pm 0.02 \mathrm{hPa}$. As the deep convective cloud begins developing ( -30 to $-40 \mathrm{~min}$ ), positive pressure anomalies slowly increase to $0.1 \mathrm{hPa} 10 \mathrm{~min}$ prior to CI. The average positive anomaly sharply increases around the time of CI to $0.5-0.6 \mathrm{hPa}$ as the convective downdrafts become established.

\section{d. Correlation length scales}

To suitably observe the preconvective environment, it is important to consider the area over which a given observation may be able to provide useful information about the state. The spatial variance at the initial time is very small, but this grows during the cloud formation period and through eventual precipitation and cold pool generation. As such, it is expected that spatial extent of

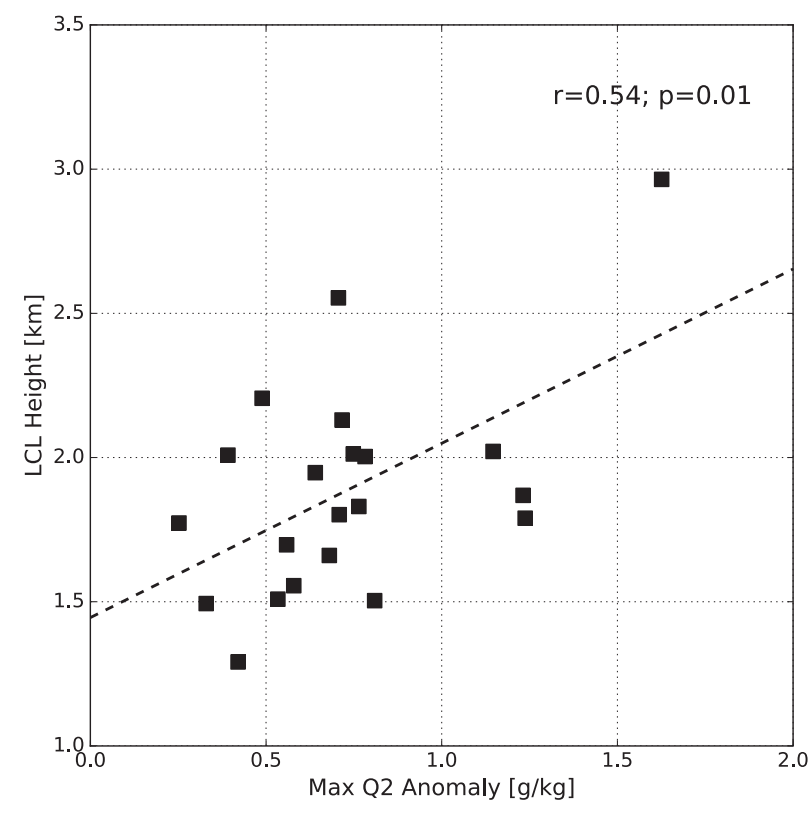

FIG. 7. The maximum positive composite $\mathrm{Q} 2$ anomaly during the 30 min prior to CI for each environment shown in Fig. 6 (colors) vs the ensemble mean, domain-averaged LCL height $(\mathrm{km}) 30 \mathrm{~min}$ prior to CI. A linear regression is shown for these points with a correlation coefficient of 0.54 and $p$ value of 0.01 for a null hypothesis of the slope equaling zero in the regression.

an observation's utility will vary over time. Furthermore, this area may vary among different variables, indicating that particular observation types may be more efficient at providing state information over a larger area with fewer observations.

For each environment simulated, we use the 25-member ensemble for that environment to compute the ensemble correlation magnitudes as they evolve throughout the simulations. Specifically, at every $5 \mathrm{~min}$ during the simulations, for T2, Q2, V10, and PSFC we compute the ensemble correlation between each variable at every grid point and the same variable at all surrounding grid points as a function of distance across the entire domain. This is averaged across all points in the domain to derive an average decorrelation length scale at a given time. We choose a correlation magnitude of 0.2 as a threshold for decorrelation, as estimates of statistical significance using a standard $t$ test indicated that, on average, this was the largest correlation magnitude that maintained statistical significance with $95 \%$ confidence. ${ }^{1}$ We also examined cross correlations

\footnotetext{
${ }^{1}$ We note that using correlations at all $N$ points in the domain has fewer degrees of freedom (DOF) than the total number of points in the domain, and compute our significance assuming $\mathrm{DOF}=0.2 \mathrm{~N}$, which is more appropriate given the relatively short correlation length scales that we observe.
} 
between different combinations of these variables (not shown), and while these correlations do exist they were generally smaller in spatial scale and lower in magnitude than correlations between the same variable. For two environments we also conducted limited simulations at higher $(200 \mathrm{~m})$ resolution and found that the correlation length scales were similar (not shown).

Figure 8 shows the evolution of these decorrelation length scales as a function of time relative to the ensemble-averaged time of the first CI event in each environment. For T2, Q2, and V10, the mean correlation length scale is $3-4 \mathrm{~km}$ throughout the preconvective period, ranging from 2 to $7 \mathrm{~km}$ depending on the environment. There is a slow decrease in the length scale as the time of CI approaches, reaching a minimum as clouds begin to populate the domain during the $100 \mathrm{~min}$ before CI. Correlation length scales increase for all three variables after precipitation begins, as the domain begins to be dominated by large, well-defined cold pools. Surface temperature increases in correlation length scale about $90 \mathrm{~min}$ before $\mathrm{CI}$, which appears related to the cloud-shadowing effect, separating the domain into one of two regimes (underneath a cloud or not), similar to cold pools. We note that, at any given time, there is some variability in the correlation length scale depending on the point chosen and its proximity to coherent features like cold pools, but the mean correlation length scale gives the best estimate for the potential spatial scale of observational impacts.

Surface pressure correlation length scales (Fig. 8, lower right) are generally broader in extent throughout the simulations, with mean length scales between 4 and $7 \mathrm{~km}$ but a range of $2-12 \mathrm{~km}$ in the hours prior to CI. Like T2, PSFC also sees a marked increase in correlation length scale in the $100 \mathrm{~min}$ prior to CI, which is likely related to the development and deepening of clouds. We note that this coincides with the growing positive pressure anomaly seen in Figs. 4 and 6.

\section{e. Vertical correlations}

Here we briefly consider the ability of surface observations to constrain not just the boundary layer, but features of the free atmosphere. As noted above, the largest surface anomalies we detect-the cold anomalies in T2-appear closely related to the presence of clouds above. This suggests that, absent other larger-scale variability, in the time prior to CI there could exist a correlation between surface cold anomalies and water vapor or cloud water above. Figure 9 shows the domainaveraged ensemble correlation between $\mathrm{T} 2$ and the sum of water vapor and cloud water $(\mathrm{Qv}+\mathrm{Qc})$ in the column above each point as a function of time for the same
8 August 2012 KBMX ensemble as in Fig. 4. Note that in this ensemble, the average time when precipitation first occurred is at $520 \mathrm{~min}$ (about $8.6 \mathrm{~h}$ ) into the simulation, or about 1540 local time.

The growth of the CBL is clearly seen as the depth of nonzero correlations increases during the first $360 \mathrm{~min}$ of the simulations. During this time, horizontal bands of higher (lower) correlation just above the boundary layer correspond reasonably well with layers of higher (lower) relative humidity in the initial sounding profile (Fig. 9, left panel). During the latter half of this period, the horizontal bands appear to transition to vertical bands of correlation within the boundary layer as time progresses. As the CBL deepens in the ensemble, the estimated depth of the boundary layer is positively correlated with the temperature at the surface (not shown). Thus, areas of warmer surface temperatures tend to have a locally deeper CBL, which entrains air from a higher height. In the presence of a varying vertical profile of moisture, the humidity of the entrained air will vary depending on the depth of the CBL. In our idealized setting, this illustrates how observations of surface temperature may provide information about moisture within and just above the CBL. At present, it is unclear whether more realistic simulations that include larger-scale variability can be constrained enough to fully realize these correlations.

After $360 \mathrm{~min}$, clouds begin forming in the ensemble and the character of the correlation patterns in Fig. 9 changes. At 360-400 min, rapidly deepening cumulus clouds appear in the simulations. The area of negative correlation between surface temperature and moisture aloft deepens with cloud development from a base at $4 \mathrm{~km}$ up to over $14 \mathrm{~km}$ during $400-600 \mathrm{~min}$, affirming the connection between a cold, "shadowed" temperature at the surface and higher moisture in a cloud aloft over a relatively deep layer. Below $4 \mathrm{~km}$, cold pools become the dominant feature after the onset of precipitation (here, again, at $520 \mathrm{~min}$ ), showing a positive correlation between colder air at the surface and drier (with a lower saturation mixing ratio than the ambient environment mixing ratio) downdraft air populating the boundary layer. These correlation patterns illustrate the potential for surface observations to inform the moisture field throughout the CI process over a large depth of the troposphere.

Again, it is unclear whether more realistic simulations may have strong enough constraints on the background environment and larger-scale variability to realize correlations of the magnitudes shown here. However, in environments where local, boundary layer variability exerts a primary control on CI predictability-environments 
$\mathrm{T} 2$
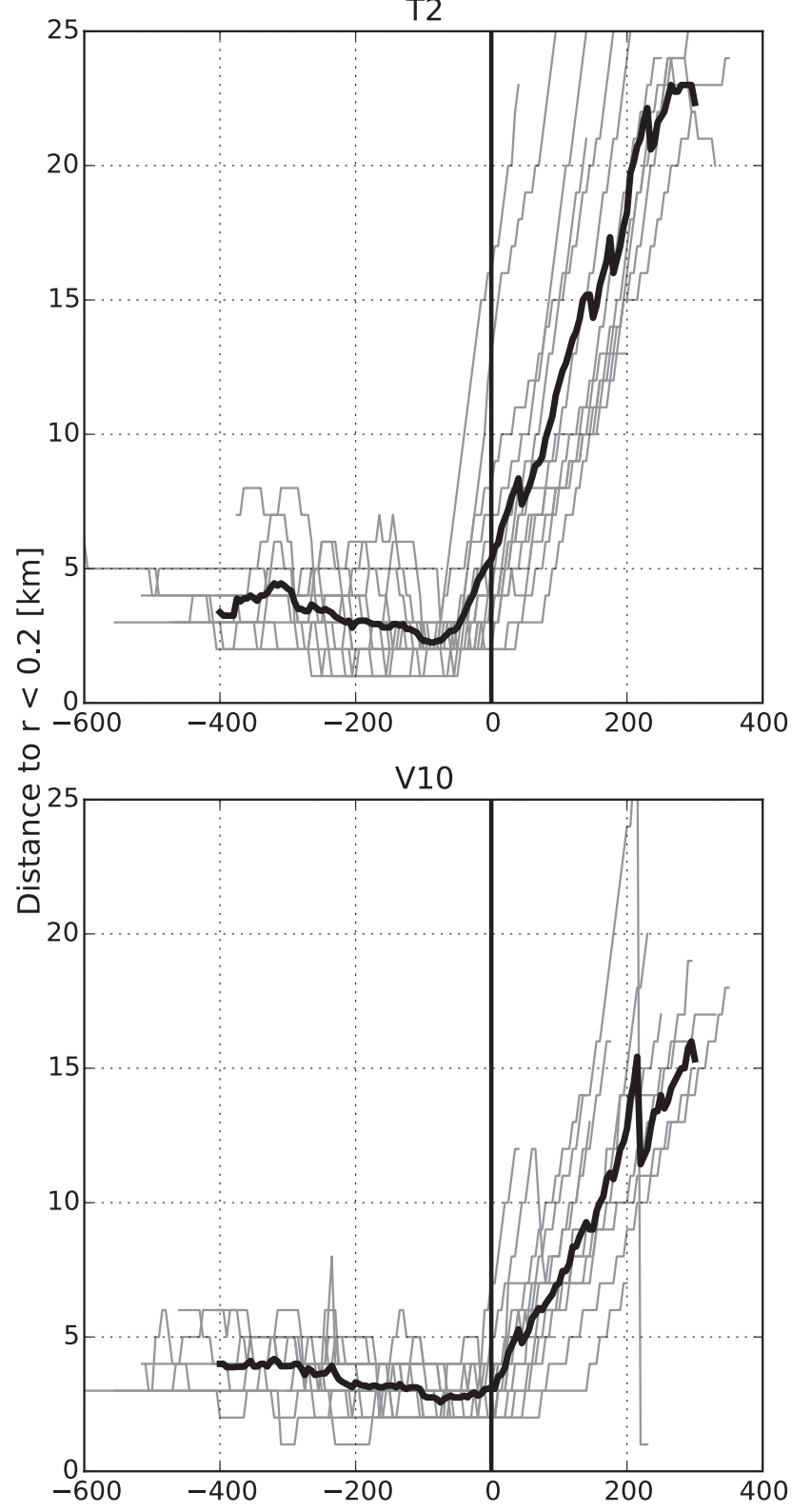
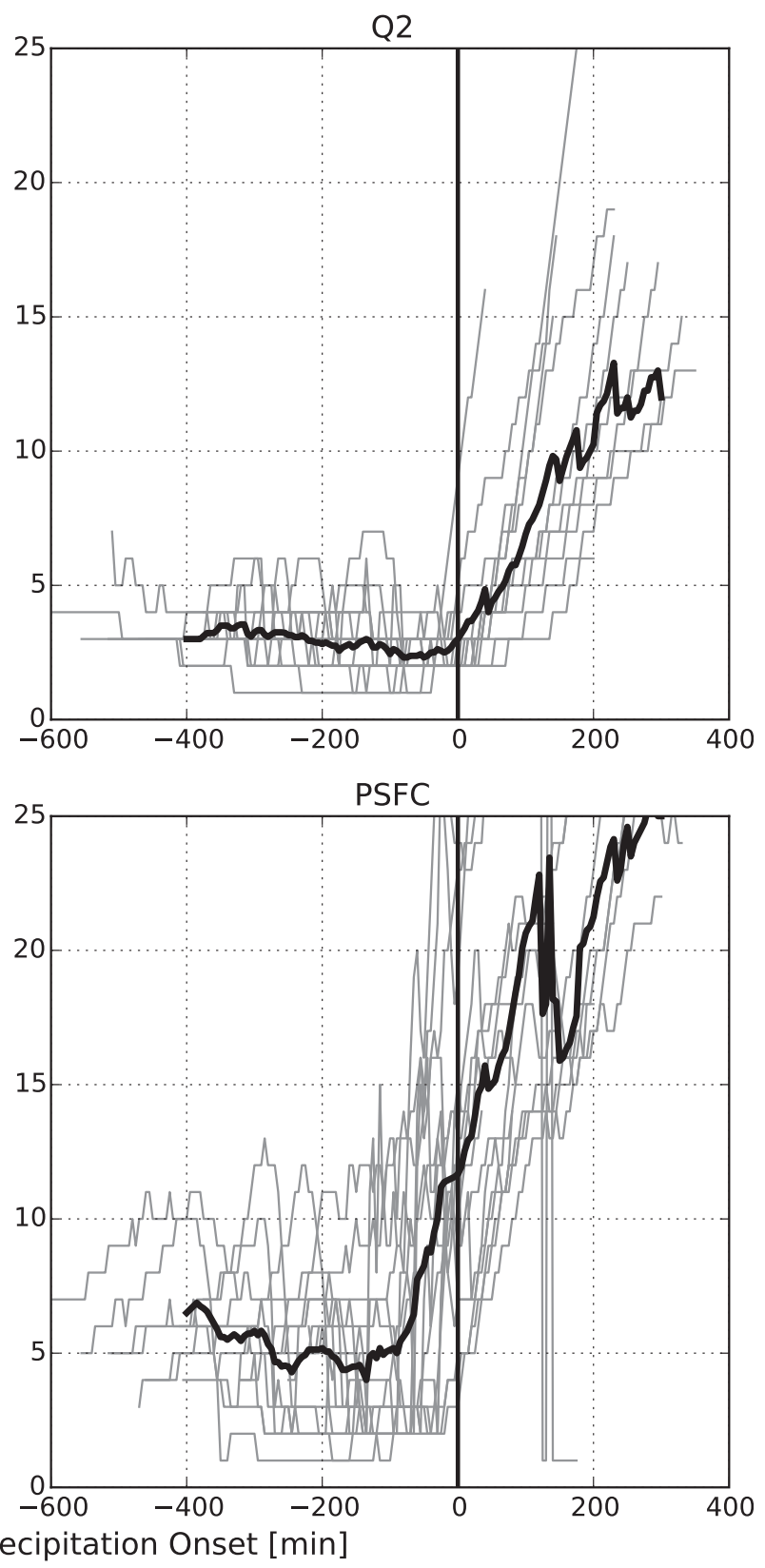

FIG. 8. Average distance from a given point where the magnitude of the ensemble correlation to that point decreases to below 0.2 as a function of time relative to the average initial time of CI. Each environment simulation is shown in gray with distances binned to the nearest kilometer. The mean decorrelation distance is shown in solid black. The average time of the first precipitating storm (time 0 ) is highlighted by the vertical black line.

where $\mathrm{CI}$ is much harder to predict-these correlations illustrate the potential for dense surface observations to constrain variability in moisture throughout a deep layer of the troposphere and to capture the initiation and maturation of convective storms, extending predictability. Further discussion of this finding, including the limitations of these idealized experiments, follows in section 6 .

\section{Implications for surface observations of CI}

a. Comparing observation effectiveness for buoyancy and convergence

As noted in section 1, there remains an open question about which surface variable is more important for constraining CI, particularly with respect to temperature and moisture, which primarily describe the buoyancy of 

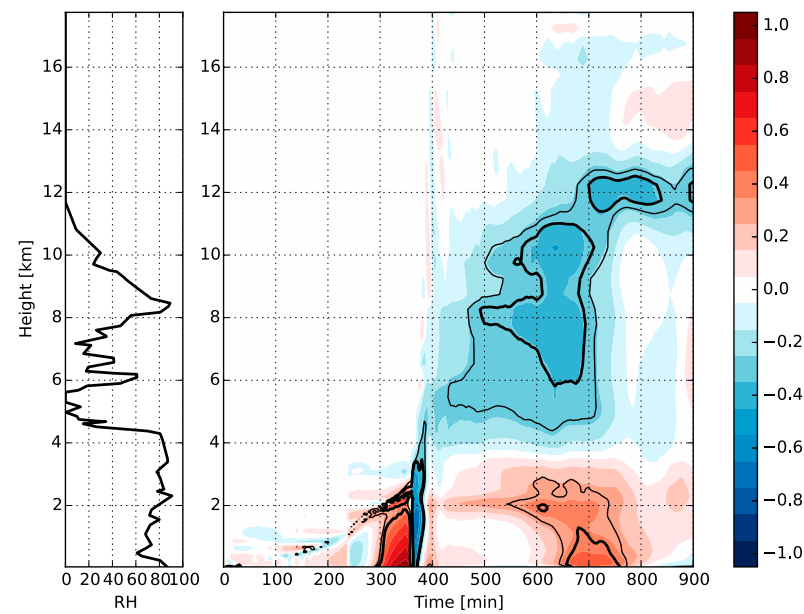

FIG. 9. (left) Relative humidity as a function of height and (right) average ensemble correlation of surface temperature to the sum of water vapor and cloud water $(\mathrm{Qv}+\mathrm{Qc})$ in the column above as a function of time ( $x$ axis) and height ( $y$ axis) for the 8 Aug 2014 KBMX environment. Thin (thick) black contours show correlation $t$-test statistical significance at the $90 \%$ (95\%) confidence level.

parcels. From Fig. 6, the largest anomalies relative to the background variance occur in $\mathrm{T} 2$, which are also the earliest to appear in composites and the largest in spatial extent, more than $1 \mathrm{~h}$ before CI. Moisture anomaly magnitudes are highly dependent on the environment, suggesting in some cases local moisture variability may be more important (e.g., when LCL levels are high) for discriminating locations of CI. The average maximum moisture magnitudes seen in the preconvective environment $\left(0.5 \mathrm{~g} \mathrm{~kg}^{-1}\right)$ are consistent with observational studies (Crook 1996; Fabry 2006; Martin and Xue 2006) that suggest moisture variability of $1 \mathrm{~g} \mathrm{~kg}^{-1}$ or less.

Surface winds and pressure largely serve as potential indicators of convergence as a precursor for CI. A convergent wind signal is apparent in all the composited pre-CI environments, with the maximum average velocity extrema $\left( \pm 1 \mathrm{~m} \mathrm{~s}^{-1}\right)$ consistent with previous observational studies of preconvective environments (Weckwerth et al. 1999; Arnott et al. 2006). The surface pressure anomalies are extremely weak $(<0.1 \mathrm{hPa})$, which would require very well-calibrated and sensitive instruments to detect. Thus, it appears surface wind observations would be most beneficial for describing convergence prior to CI.

\section{b. Observation accuracy and density requirements}

To further consider the practical effectiveness of surface observations to capture the anomalies described above, we consider these anomalies in the context of the data assimilation problem. Specifically, we compute the number of independent observations that would be required to produce an analysis with an error variance sufficiently small to resolve an anomaly of a given magnitude with a specified degree of confidence. We employ the Kalman filter update equation to update the analysis error variance upon assimilating a single observation. Since the number of observations that will be required is not known a priori, we use the ensemble square root filter variant, which is formulated for serial observation processing (Whitaker and Hamill 2002). For each single, scalar variable that is directly observed, the analysis error variance is given by

$$
\sigma_{a}^{2}=\left[1-\beta \sigma_{b}^{2}\left(\sigma_{b}^{2}+r^{2}\right)^{-1}\right] \sigma_{b}^{2},
$$

where $\sigma_{a}^{2}$ is the analysis error variance, $\sigma_{b}^{2}$ is the background (prior) error variance, $r^{2}$ is the observation error variance, and $\beta$ is the "square root factor," given below, that accounts for observation sampling error and is a function of $r^{2}$ and $\sigma_{b}^{2}$ (Whitaker and Hamill 2002):

$$
\beta=\left[1+\sqrt{\frac{r^{2}}{\sigma_{b}^{2}+r^{2}}}\right]^{-1} .
$$

For each observation assimilated, the analysis error variance is reduced. We iterate this equation for multiple independent observations with uncorrelated errors until a target variance is reached.

Figure 10 illustrates the objective of this exercise. The variance of a background distribution ("prior") is reduced by serial assimilation of observations until the analysis error variance ("posterior"), which is also the ensemble variance, is small enough to constrain the error in the ensemble mean with a specified degree of confidence to within $\pm n$. The number of independent observations required to achieve this is recorded. Here, confidence levels of $70 \%$ and $90 \%$ will be considered.

There are three free parameters to consider in this analysis: the observation error variance $r^{2}$, the background (prior) error variance $\sigma_{b}^{2}$, and the magnitude of the anomaly to resolve $n$. Figure 11 illustrates the observation counts required to resolve a range of different anomaly magnitudes. In Figs. 11a and 11b, several observation error variances are examined while assuming an initial background error variance $\left(\sigma_{b}^{2}\right)$ of 1 unit $^{2}$ in all cases. In Figs. 11c and 11d, a range of different initial background error variances are considered while keeping a fixed observation error variance of 1 unit $^{2}$.

In most operational data assimilation systems, surface observation error variances are prescribed to be about 1 unit $^{2}$ for T2 (K), PSFC (hPa), 10-m wind $\left(\mathrm{m} \mathrm{s}^{-1}\right)$, and Q2 $\left(\mathrm{g} \mathrm{kg}^{-1}\right.$ ) (e.g., Burton 2014; Hu et al. 2013). We consider an observation error variance of $1 \mathrm{unit}^{2}$ in the top row of Fig. 11a and in all of Figs. 11c and 11d. Recall the mean positive preconvective environment $\mathrm{T} 2$ anomalies of 


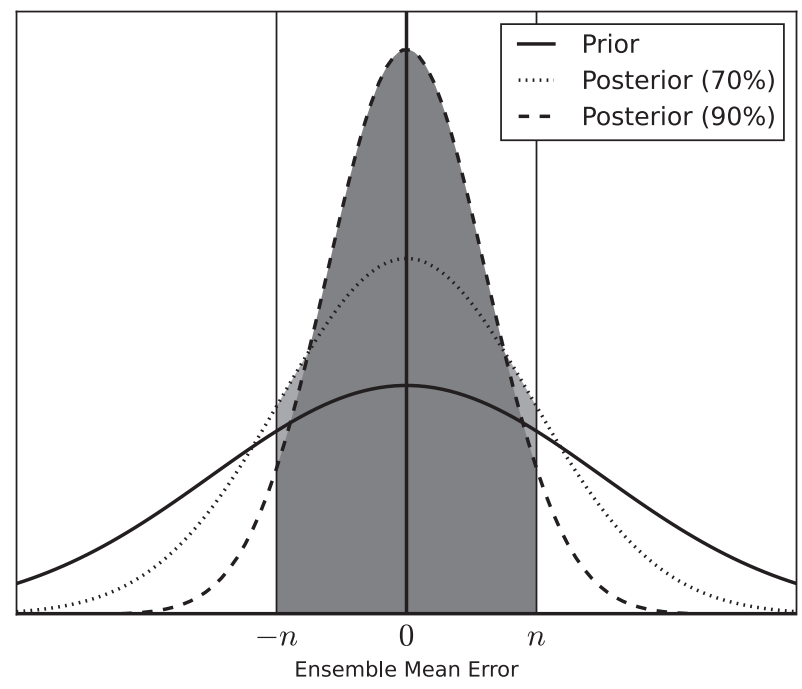

FIG. 10. Schematic of error variance reduction by observation assimilation. The ensemble mean error is shown on the $x$ axis. The variance of a background distribution (prior) is reduced by assimilating observations until the magnitude of the error in the ensemble mean is constrained to be less than $n$ with $70 \%$ (posterior $70 \%$ ) or $90 \%$ (posterior $90 \%$ ) confidence.

0.5-K magnitude shown in Fig. 6. Assuming an initial background error variance of $1 \mathrm{~K}^{2}$ and an observation error variance of $1 \mathrm{~K}^{2}$ (Fig. 11c, top row), for variations of $0.5 \mathrm{~K}$ to be resolved with $90 \%$ confidence, approximately 15 independent observations are required (topright corner). However, to resolve with only $70 \%$ confidence, only one observation is required (Fig. 11d). By reducing the background error variance to $<0.2 \mathrm{~K}^{2}$, only 1-4 observations would be required to resolve this anomaly with $90 \%$ confidence (bottom-right corner, Fig. 11c). This indicates that it is likely to be correlation length scale and not observation error that would most constrain the number of temperature observations required. For $10-\mathrm{m}$ wind anomalies of $1 \mathrm{~m} \mathrm{~s}^{-1}$, a similar conclusion may be drawn.

However, for Q2 and PSFC, the preconvective anomaly magnitudes are smaller $\left(0.25 \mathrm{~g} \mathrm{~kg}^{-1} \mathrm{Q} 2 ;<0.1-\mathrm{hPa}\right.$ PSFC), and with the observation error variance suggested above $\left(1\right.$ unit $\left.^{2}\right)$ a much larger number of independent observations would be required to resolve these magnitudes. In particular, for PSFC, over 300 (100) observations would be required to resolve these magnitudes with $90 \%(70 \%)$ confidence (Fig. 11). Though PSFC has a slightly longer correlation length scale (Fig. 8), an observation error variance of $1 \mathrm{hPa}^{2}$ is too large for even an extremely dense PSFC observing network to resolve anomalies of this magnitude. In Figs. 11a and 11b, we see that even reducing the observation error variance to $0.1 \mathrm{hPa}^{2}$ would still require about 40 (8) independent observations to resolve a 0.1 -hPa anomaly with $90 \%$ (70\%) confidence.
In general, as the initial background error variance is reduced (Figs. 11c and 11d), the required number of independent observations becomes more tractable. One way to maintain a small background error variance would be to cycle an ensemble forecast more frequently, not allowing time for ensemble member forecasts to diverge far from observations (e.g., Sobash and Stensrud 2015). This is one way to possibly make PSFC and Q2 observations more relevant to storm-scale assimilation in the pre-CI environment.

It is also possible that these observation error variances may be overestimates. Hacker and RostkierEdelstein (2007) estimate observation error variance from instruments at the Oklahoma Atmospheric Radiation Measurement (ARM) site and suggest temperature and moisture observation error variances may be as small as $0.08 \mathrm{~K}^{2}$ and $0.077 \mathrm{~g}^{2} \mathrm{~kg}^{-2}$, respectively. Even barometric pressure measurements from modern smartphone sensors have a specified relative error variance of $0.04 \mathrm{hPa}^{2}$ (Mass and Madaus 2014). While sensor error is only one component of the total observation error, it suggests that a network of well-calibrated barometers may be capable of resolving smaller anomalies than presently assumed.

We also note that this analysis assumes that the model background estimate and the observation values are unbiased, which is not true for most model forecasts and observations. For instance, Hu et al. (2010) find that WRF simulations tend to consistently underpredict temperature and overpredict moisture over the south-central United States, though the biases do vary depending on the specifics of the PBL scheme used, and this method does not take these biases into account. Nevertheless, this idealized perspective is still useful for evaluating observation effectiveness even within a "perfect" scenario.

\section{Summary and conclusions}

In this study, we examined isolated, locally driven CI in a variety of different environments with idealized ensemble simulations. A CI-relative compositing method revealed coherent features in all surface fields examined throughout the CI process. Warm 2-m temperature anomalies were present from $90-170 \mathrm{~min}$ prior to the onset of precipitation, with moisture, wind, and pressure anomalies also detectable about $1 \mathrm{~h}$ before CI. Wind and moisture anomalies were found to be consistent with observational studies of preconvective environments. The strongest anomalies prior to CI were related to cloud shadowing, creating a cold anomaly of $1-2 \mathrm{~K}$ as the convective cloud deepens. Surface pressure anomalies remained relatively weak $(<0.1 \mathrm{hPa})$ throughout the $\mathrm{CI}$ process. 

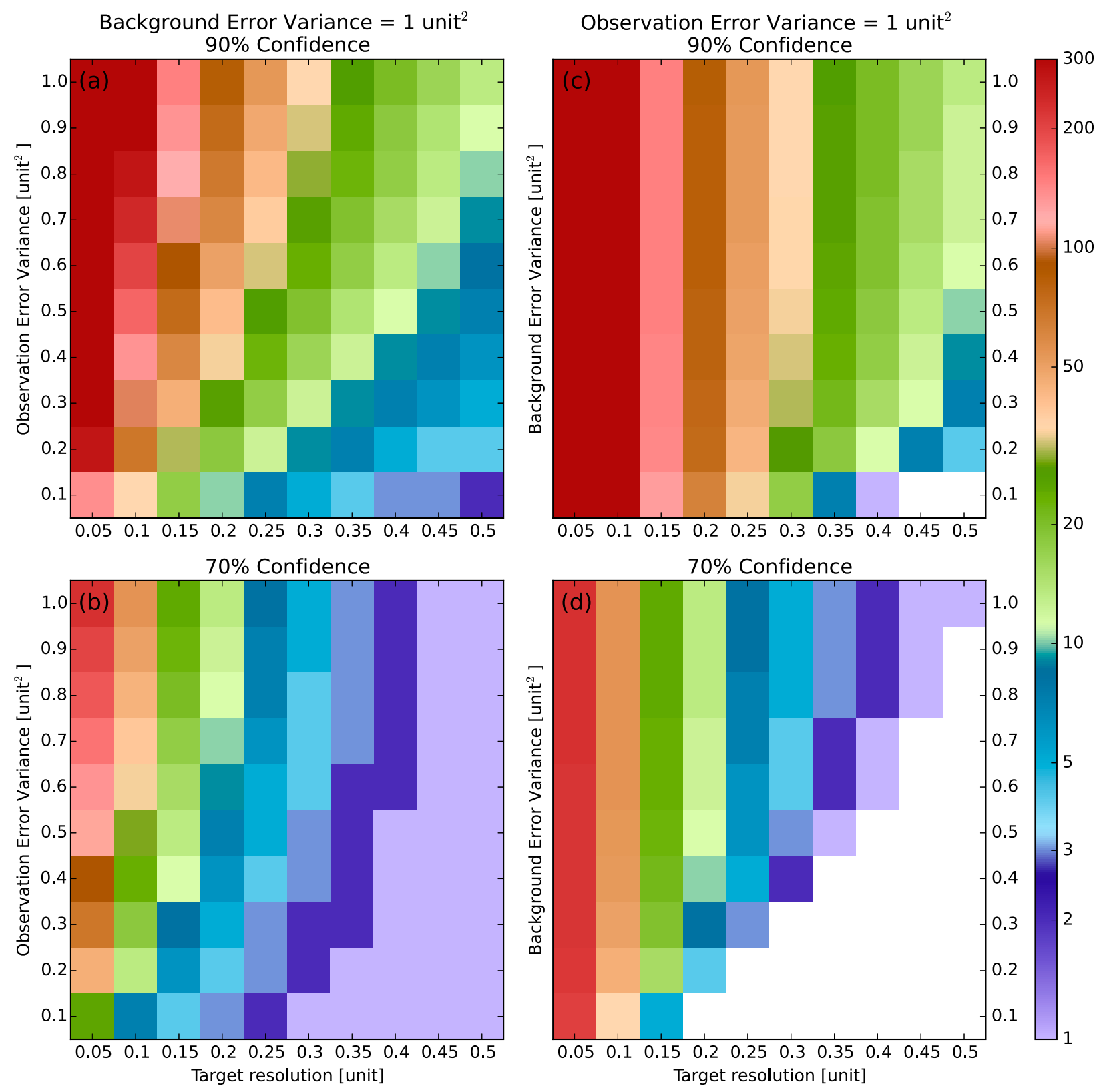

FIG. 11. Number of independent observations required to reduce the analysis error variance such that it resolves a given anomaly magnitude ( $x$ axes). (a),(b) A range of observation error variances are examined ( $y$ axes) with an initial background error variance of 1 unit $^{2}$. (c),(d) A range of initial background error variances are examined ( $y$ axes) with the observation error variance fixed at 1 unit $^{2}$. The number of observations is computed to resolve the given anomaly magnitudes with (a),(c) $90 \%$ confidence and (b),(d) $70 \%$ confidence. White regions already have a background error variance small enough to resolve the specified anomaly magnitude with the expected confidence prior to any observation assimilation.

In general, all surface fields (except surface pressure) showed horizontal correlation length scales of 3-4 km prior to $\mathrm{CI}$, with surface pressure having a slightly longer length scale of 4-7 km. These suggest an upper bound of about one observation every $5 \mathrm{~km}$ for a storm-scale observing network, neglecting temporal correlations. Strong vertical correlations were also observed, particularly between surface temperature anomalies and moisture anomalies aloft. This further suggests that surface observations may help constrain storm-scale features throughout the troposphere, given a well-constrained background environment.

The feasibility of resolving pre-CI surface anomalies was examined in the context of variance reduction 
through data assimilation. We show that the necessary temperature and wind anomalies would most easily be resolved using current assumptions about observation error variance, given a realistically feasible observation density. Capturing moisture and pressure anomalies would require either an extremely dense network, frequent assimilation cycling, or observations with much smaller error variances than currently assumed.

One limitation to these results is the use of a single PBL scheme. The surface fields examined here are chiefly outputs of this scheme, and using a different scheme may yield different results. However, Hacker and RostkierEdelstein (2007) suggest that the ability of surface observations to constrain the PBL is not dependent on the PBL parameterization used, given sufficiently frequent assimilation cycling. Future studies may wish to compare these results with other PBL schemes; here we were limited by the single YSU PBL option available in the CM1 model.

In all environments simulated, the same pattern of anomalies is present during the hours preceding CI, suggesting that there are indeed robust surface features that an appropriate observing network could sample. While these results are encouraging, they must be considered within the context of this idealized modeling setup. For instance, though there are strong patterns of vertical correlation between surface temperature and the moisture in the troposphere aloft (section 4e), much of this correlation strength is likely due to the nearly identical vertical moisture profiles in all ensemble members without synoptic and broad mesoscale variability. It is unlikely that a real-world ensemble would have such a tightly constrained background environment, and that could lessen the correlation magnitude. Indeed, the role of entrainment in promoting or inhibiting CI is largely ignored in this experiment, as clouds forming in all ensemble members encounter similar conditions aloft. Likewise, the horizontal correlation length scales in more realistic settings could vary more substantially than shown here due to inhomogeneities in the land surface characteristics not represented here.

The correlation length scale evolution and the small variance in the anomaly growth time scale is also likely due to all ensemble members producing clouds and eventually precipitation at about the same time; in a real-world ensemble with greater variance there is likely to be more diversity in the time and spatial locations where $\mathrm{CI}$ is favorable. However, these results suggest a more rigorous condition for successful storm-scale CI prediction: even with a tightly constrained background state, the exact location and timing of CI still is subject to the small-scale variability seen here.
While this work has considered simulations of multiple environments to specifically characterize the variations in surface fields surrounding successful convective initiation, our approach provides a framework for further examination of the CI process. For instance, the storm tracking algorithm used here could be modified to track developing cloud objects and identify specific differences between clouds that deepen and produce precipitation and those that fail to do so within an environment with little to no mesoscale or synoptic-scale variability. This could suggest additional observation platforms that may be useful in successfully discriminating CI successes and failures on the storm scale.

Finally, we note that this study does not explicitly connect improved resolution of surface features to actual improved forecasts of CI. Important questions remain about translating better surface representation to CI forecast improvement, particularly when faced with a more varied background environment and complicating factors such as entrainment. Future work involving observing system simulation experiments (OSSEs) with these and other simulations is planned to test hypothesized observation densities as suggested by this research, examining whether resolving these anomalies actually leads to improved forecasts of CI.

Acknowledgments. This work was supported through NOAA CSTAR Award NA13NWS4680006. We greatly appreciate comments provided by Cliff Mass that helped refine this manuscript. We would also like to thank Paul Markowski for discussions about the effects of cloud shadowing that greatly contributed to our analysis.

\section{REFERENCES}

Allan, D., T. A. Caswell, N. Keim, F. Boulogne, R. W. Perry, and L. Uieda, 2014: trackpy: Trackpy v0.2.4. doi:10.5281/ zenodo.12255. [Available online at http://github.com/ soft-matter/trackpy.]

Arnott, N. R., Y. P. Richardson, J. M. Wurman, and E. M. Rasmussen, 2006: Relationship between a weakening cold front, misocyclones, and cloud development on 10 June 2002 during IHOP. Mon. Wea. Rev., 134, 311-335, doi:10.1175/ MWR3065.1.

Benjamin, S. G., and T. N. Carlson, 1986: Some effects of surface heating and topography on the regional severe storm environment. Part I: Three-dimensional simulations. Mon. Wea. Rev., 114, 307-329, doi:10.1175/1520-0493(1986)114<0307: SEOSHA $>2.0 . \mathrm{CO} ; 2$.

Bluestein, H. B., E. W. McCaul, G. P. Byrd, and G. R. Woodall, 1988: Mobile sounding observations of a tornadic storm near the dryline: The Canadian, Texas, storm of 7 May 1986. Mon. Wea. Rev., 116, 1790-1804, doi:10.1175/1520-0493(1988)116<1790: MSOOAT $>2.0 . \mathrm{CO} ; 2$.

Brooks, H. E., C. A. Doswell, and R. A. Maddox, 1992: On the use of mesoscale and cloud-scale models in operational 
forecasting. Wea. Forecasting, 7, 120-132, doi:10.1175/ 1520-0434(1992)007<0120:OTUOMA > 2.0.CO;2.

Bryan, G. H., and J. M. Fritsch, 2002: A benchmark simulation for moist nonhydrostatic numerical models. Mon. Wea. Rev., 130, 2917-2928, doi:10.1175/1520-0493(2002)130<2917:ABSFMN > 2.0.CO;2.

— J. C. Wyngaard, and J. M. Fritsch, 2003: Resolution requirements for the simulation of deep moist convection. Mon. Wea. Rev., 131, 2394-2416, doi:10.1175/1520-0493(2003)131<2394: RRFTSO $>2.0 . \mathrm{CO} ; 2$.

Burton, P., 2014: IFS documention-Cy40r1. Part I: Observations. Tech. Rep., ECMWF, 76 pp. [Available online at http://www.ecmwf.int/ sites/default/files/IFS_CY40R1_Part1.pdf.]

Childs, P. P., A. L. Qureshi, S. Raman, K. Alapaty, R. Ellis, R. Boyles, and D. Niyogi, 2006: Simulation of convective initiation during IHOP 2002 using the flux-adjusting surface data assimilation system (FASDAS). Mon. Wea. Rev., 134, 134148, doi:10.1175/MWR3064.1.

Chou, M. D., and M. J. Suarez, 1999: A solar radiation parameterization for atmospheric studies. Tech. Rep. NASA/TM1999-10460, NASA, 38 pp.

— , and - 2001: A thermal infrared radiation parameterization for atmospheric studies. Tech. Rep. NASA/TM-2001104606, NASA, 55 pp.

Clark, D. B., C. M. Taylor, and A. J. Thorpe, 2004: Feedback between the land surface and rainfall at convective length scales. J. Hydrometeor., 5, 625-639, doi:10.1175/1525-7541(2004)005<0625: FBTLSA $>2.0 . \mathrm{CO} ; 2$.

Crook, N. A., 1996: Sensitivity of moist convection forced by boundary layer processes to low-level thermodynamic fields. Mon. Wea. Rev., 124, 1767-1785, doi:10.1175/1520-0493(1996)124<1767: $\mathrm{SOMCFB}>2.0 . \mathrm{CO} ; 2$.

Dabberdt, W. F., and T. W. Schlatter, 1996: Research opportunities from emerging atmospheric observing and modeling capabilities. Bull. Amer. Meteor. Soc., 77, 305-323, doi:10.1175/ 1520-0477(1996)077<0305:ROFEAO > 2.0.CO;2.

— , and Coauthors, 2005: Multifunctional mesoscale observing networks. Bull. Amer. Meteor. Soc., 86, 961-982, doi:10.1175/ BAMS-86-7-961.

Davis, C., B. Brown, and R. Bullock, 2006: Object-based verification of precipitation forecasts. Part I: Methodology and application to mesoscale rain areas. Mon. Wea. Rev., 134, 17721784, doi:10.1175/MWR3145.1.

Dong, J., M. Xue, and K. Droegemeier, 2011: The analysis and impact of simulated high-resolution surface observations in addition to radar data for convective storms with an ensemble Kalman filter. Meteor. Atmos. Phys., 112, 41-61, doi:10.1007/ s00703-011-0130-3.

Droegemeier, K. K., and R. B. Wilhelmson, 1985: Threedimensional numerical modeling of convection produced by interacting thunderstorm outflows. Part I: Control simulation and low-level moisture variations. J. Atmos. Sci., 42, 2381-2403, doi:10.1175/1520-0469(1985)042<2381:TDNMOC >2.0.CO;2.

Durran, D., and J. Weyn, 2016: Thunderstorms do not get butterflies. Bull. Amer. Meteor. Soc., 97, 237-243, doi:10.1175/ BAMS-D-15-00070.1.

Fabry, F., 2006: The spatial variability of moisture in the boundary layer and its effect on convection initiation: Project-long characterization. Mon. Wea. Rev., 134, 79-91, doi:10.1175/ MWR3055.1.

Fowle, M. A., and P. J. Roebber, 2003: Short-range (0-48 h) numerical prediction of convective occurrence, mode, and location. Wea. Forecasting, 18, 782-794, doi:10.1175/1520-0434(2003)018<0782: SHNPOC $>2.0 . C O ; 2$.
Gallus, W. A., J. Correia, and I. Jankov, 2005: The 4 June 1999 derecho event: A particularly difficult challenge for numerical weather prediction. Wea. Forecasting, 20, 705-728, doi:10.1175/WAF883.1.

Hacker, J. P., and D. Rostkier-Edelstein, 2007: PBL state estimation with surface observations, a column model, and an ensemble filter. Mon. Wea. Rev., 135, 2958-2972, doi:10.1175/MWR3443.1.

Hohenegger, C., and C. Schaar, 2007: Atmospheric predictability at synoptic versus cloud-resolving scales. Bull. Amer. Meteor. Soc., 88, 1783-1793, doi:10.1175/BAMS-88-11-1783.

Holt, T. R., D. Niyogi, F. Chen, K. Manning, M. A. LeMone, and A. Qureshi, 2006: Effect of land-atmosphere interactions on the IHOP 24-25 May 2002 convection case. Mon. Wea. Rev., 134, 113-133, doi:10.1175/MWR3057.1.

Hong, S., Y. Noh, and J. Dudhia, 2006: A new vertical diffusion package with an explicit treatment of entrainment processes. Mon. Wea. Rev., 134, 2318-2341, doi:10.1175/MWR3199.1.

Hu, M., H. Shao, D. Stark, and K. Newman, 2013: Gridpoint statistical interpolation (GSI) version 3.2 user's guide. Tech. Rep. (error variances obtained from file nam_errtable.r3dv), 187 pp. [Available online at http://www.dtcenter.org/com-GSI/ users/docs/users_guide/GSIUserGuide_v3.2.pdf.]

$\mathrm{Hu}$, X., J. Nielsen-Gammon, and F. Zhang, 2010: Evaluation of three planetary boundary layer schemes in the WRF model. J. Appl. Meteor. Climatol., 49, 1831-1844, doi:10.1175/ 2010JAMC2432.1.

Jonker, H. J. J., P. G. Duynkerke, and J. W. M. Cuijpers, 1999: Mesoscale fluctuations in scalars generated by boundary layer convection. J. Atmos. Sci., 56, 801-808, doi:10.1175/ 1520-0469(1999)056<0801:MFISGB >2.0.CO;2.

Kang, S., and G. H. Bryan, 2011: A large-eddy simulation study of moist convection initiation over heterogeneous surface fluxes. Mon. Wea. Rev., 139, 2901-2917, doi:10.1175/ MWR-D-10-05037.1.

Koch, S. E., 1984: The role of an apparent mesoscale frontogenetic circulation in squall line initiation. Mon. Wea. Rev., 112, 2090-2111, doi:10.1175/1520-0493(1984)112<2090: TROAAM $>2.0 . \mathrm{CO} ; 2$.

Liu, H., and M. Xue, 2008: Prediction of convective initiation and storm evolution on 12 June 2002 during IHOP 2002. Part I: Control simulation and sensitivity experiments. Mon. Wea. Rev., 136, 2261-2282, doi:10.1175/2007MWR2161.1.

Lohou, F., and E. G. Patton, 2014: Surface energy balance and buoyancy response to shallow cumulus shading. J. Atmos. Sci., 71, 665-682, doi:10.1175/JAS-D-13-0145.1.

Madaus, L. E., G. J. Hakim, and C. F. Mass, 2014: Utility of dense pressure observations for improving mesoscale analyses and forecasts. Mon. Wea. Rev., 142, 2398-2413, doi:10.1175/ MWR-D-13-00269.1.

Martin, W. J., and M. Xue, 2006: Sensitivity analysis of convection of the 24 May 2002 IHOP case using very large ensembles. Mon. Wea. Rev., 134, 192-207, doi:10.1175/MWR3061.1.

Mass, C. F., and L. E. Madaus, 2014: Surface pressure observations from smartphones: A potential revolution for high-resolution weather prediction? Bull. Amer. Meteor. Soc., 95, 1343-1349, doi:10.1175/BAMS-D-13-00188.1.

_ D. Ovens, K. Westrick, and B. A. Colle, 2002: Does increasing horizontal resolution produce more skillful forecasts? Bull. Amer. Meteor. Soc., 83, 407-430, doi:10.1175/1520-0477(2002)083<0407: DIHRPM>2.3.CO;2.

Nowotarski, C. J., P. M. Markowski, Y. P. Richardson, and G. H. Bryan, 2014: Properties of a simulated convective boundary layer in an idealized supercell thunderstorm 
environment. Mon. Wea. Rev., 142, 3955-3976, doi:10.1175/ MWR-D-13-00349.1.

Owen, J., 1966: A study of thunderstorm formation along dry lines. J. Appl. Meteor., 5, 58-63, doi:10.1175/1520-0450(1966)005<0058: ASOTFA $>2.0 . \mathrm{CO} ; 2$.

Rieck, M., C. Hohenegger, and P. Gentine, 2015: The effect of moist convection on thermally induced mesoscale circulations. Quart. J. Roy. Meteor. Soc., 141, 2418-2428, doi:10.1002/qj.2532.

Roebber, P. J., D. M. Schultz, and R. Romero, 2002: Synoptic regulation of the 3 May 1999 tornado outbreak. Wea. Forecasting, 17, 399-429, doi:10.1175/1520-0434(2002)017<0399: SROTMT > 2.0.CO;2.

Schwartz, C. S., and Coauthors, 2009: Next-day convectionallowing WRF model guidance: A second look at 2-km versus 4-km grid spacing. Mon. Wea. Rev., 137, 3351-3372, doi:10.1175/2009MWR2924.1.

Skamarock, W. C., 2004: Evaluating mesoscale NWP models using kinetic energy spectra. Mon. Wea. Rev., 132, 3019-3032, doi:10.1175/MWR2830.1.

- , and Coauthors, 2008: A description of the Advanced Research WRF version 3. NCAR Tech. Note NCAR/TN475+STR, 113 pp., doi:10.5065/D68S4MVH.

Snook, N., M. Xue, and Y. Jung, 2015: Multiscale EnKF assimilation of radar and conventional observations and ensemble forecasting for a tornadic mesoscale convective system. Mon. Wea. Rev., 143, 1035-1057, doi:10.1175/MWR-D-13-00262.1.

Sobash, R. A., and D. J. Stensrud, 2015: Assimilating surface mesonet observations with the EnKF to improve ensemble forecasts of convection initiation on 29 May 2012. Mon. Wea. Rev., 143, 3700-3725, doi:10.1175/MWR-D-14-00126.1.

Stensrud, D. J., and J. M. Fritsch, 1994: Mesoscale convective systems in weakly forced large-scale environments. Part III: Numerical simulations and implications for operational forecasting. Mon. Wea. Rev., 122, 2084-2104, doi:10.1175/ 1520-0493(1994)122<2084:MCSIWF>2.0.CO;2.

Sun, J., and Coauthors, 2014: Use of NWP for nowcasting convective precipitation: Recent progress and challenges. Bull. Amer. Meteor. Soc., 95, 409-426, doi:10.1175/ BAMS-D-11-00263.1.

Torn, R., and G. Romine, 2015: Sensitivity of central Oklahoma convection forecasts to upstream potential vorticity anomalies during two strongly forced cases during MPEX. Mon. Wea. Rev., 143, 4064-4087, doi:10.1175/MWR-D-15-0085.1.

VandenBerg, M. A., M. C. Coniglio, and A. J. Clark, 2014: Comparison of next-day convection-allowing forecasts of storm motion on 1- and 4-km grids. Wea. Forecasting, 29, 878-893, doi:10.1175/WAF-D-14-00011.1.

Weckwerth, T. M., and D. B. Parsons, 2006: A review of convection initiation and motivation for IHOP 2002. Mon. Wea. Rev., 134, 5-22, doi:10.1175/MWR3067.1.

— T. T. W. Horst, and J. W. Wilson, 1999: An observational study of the evolution of horizontal convective rolls. Mon. Wea. Rev., 127, 2160-2179, doi:10.1175/1520-0493(1999)127<2160: AOSOTE $>2.0 . \mathrm{CO} ; 2$.

—, L. J. Bennett, L. J. Miller, J. Van Baelen, P. Di Girolamo, A. M. Blyth, and T. J. Hertneky, 2014: An observational and modeling study of the processes leading to deep, moist convection in complex terrain. Mon. Wea. Rev., 142, 2687-2708, doi:10.1175/MWR-D-13-00216.1.

Wheatley, D. M., and D. J. Stensrud, 2010: The impact of assimilating surface pressure observations on severe weather events in a WRF mesoscale ensemble system. Mon. Wea. Rev., 138, 1673-1694, doi:10.1175/2009MWR3042.1.

Whitaker, J. S., and T. M. Hamill, 2002: Ensemble data assimilation without perturbed observations. Mon. Wea. Rev., 130, 1913-1924, doi:10.1175/1520-0493(2002)130<1913: EDAWPO $>2.0 . \mathrm{CO} ; 2$.

Yan, H., and R. A. Anthes, 1988: The effect of variations in surface moisture on mesoscale circulation. Mon. Wea. Rev., 116, 192208, doi:10.1175/1520-0493(1988)116<0192:TEOVIS > 2.0.CO;2.

Zhang, F., C. Snyder, and R. Rotunno, 2003: Effects of moist convection on mesoscale predictability. J. Atmos. Sci., 60, 1173-1185, doi:10.1175/1520-0469(2003)060<1173:EOMCOM>2.0.CO;2.

Zhang, Y., F. Zhang, D. J. Stensrud, and Z. Meng, 2015: Practical predictability of the 20 May 2013 tornadic thunderstorm event in Oklahoma: Sensitivity to synoptic timing and topographical influence. Mon. Wea. Rev., 143, 2973-2997, doi:10.1175/ MWR-D-14-00394.1.

Ziegler, C. L., T. J. Lee, and R. A. Pielke, 1997: Convective initiation at the dryline: A modeling study. Mon. Wea. Rev., 125, 1001-1026, doi:10.1175/1520-0493(1997)125<1001: CIATDA $>2.0 . \mathrm{CO} ; 2$. 